\title{
The recursive Green's function method for graphene
}

\author{
Caio H. Lewenkopf · Eduardo R. Mucciolo
}

Received: date / Accepted: date

\begin{abstract}
We describe how to apply the recursive Green's function method to the computation of electronic transport properties of graphene sheets and nanoribbons in the linear response regime. This method allows for an amenable inclusion of several disorder mechanisms at the microscopic level, as well as inhomogeneous gating, finite temperature, and, to some extend, dephasing. We present algorithms for computing the conductance, density of states, and current densities for armchair and zigzag atomic edge alignments. Several numerical results are presented to illustrate the usefulness of the method.
\end{abstract}

Keywords electronic transport - recursive Green's function method $\cdot$ graphene nanoribbons

PACS 73.23.-b 72.80.Vp 81.05.ue

\section{Introduction}

Since graphene was first produced, several synthesis strategies have been put forward. Significant progress has been made to produce better quality samples with the goal of improving their transport properties. Despite the enormous effort, we are still very far from reaching the perfect ballistic regime and disorder always plays a central role, particularly in electronic transport. Disorder appears in several different forms, being either local (such as lattice defects, edge irregularities, and surface adsorbates) or long ranged (such as charge impurities trapped in the substrate or ripples due to substrate roughness) [1].

Caio H. Lewenkopf

Instituto de Física, Universidade Federal Fluminense, Brazil

E-mail: caio@if.uff.br

Eduardo R. Mucciolo

Department of Physics, University of Central Florida, USA

E-mail:mucciolo@physics.ucf.edu
Several theoretical methods have been developed to describe electronic transport in disordered graphene [2]. The effective low-energy Dirac Hamiltonian, derived from bandstructure theory, combined with a standard diagrammatic perturbative expansion is an excellent analytical tool for giving us insight into the properties of disordered graphene [3, 4]. However, it has $\left(k_{F} \ell\right)^{-1}$ as a small expansion parameter, where $k_{F}$ stands for the Fermi wave number and $\ell$ is the electron mean free path. Thus, it describes well the conductivity in graphene at high doping, but becomes of limited use when one is interested in the physics close to the charge neutrality point, where $k_{F} \ell \ll 1$. Theoretical investigations of the that regime require instead the use of numerical methods.

Most numerical methods employed to study the transport properties of disordered graphene use an atomistic basis [2]. The few exceptions are tailor-made methods to deal with long-range disorder, where either a momentum representation [5,6] or discretized version of the Dirac equation [7,8] are used within a single-valley approximation.

For many applications, one is interested in the two- or multiple-probe conductance. For the conductance, differently from the conductivity, geometry plays an important role. The recursive Green's function (RGF) method [9] became the standard tool to compute transport properties in this case. The method is very reliable, computationally efficient, and allows for a parallel implementation [10]. It can model arbitrary geometries and efficiently addresses a variety of scattering processes within the single-particle approximation. The goal of this paper is to show how to compute electronic transport properties of graphene samples within the tightbinding approximation using the RGF method. The key element is an efficient algorithm for evaluating the singleparticle Green's function of sheets or ribbons.

The recursive method was developed by Thouless and Kirkpatrick [9] for computing the linear electronic conductance of linear atomic chains in the presence of on-site dis- 
order. The method was later generalized to two-dimensional systems in the "slice" formulation, which is the form most used nowadays [11]. Variations of the method have been introduced in the literature to treat three-dimensional [12] and multi-probe systems [13] with arbitrary geometries, see e.g. Ref. [14].

We note that other efficient, atomistic methods have been employed in recent years to study electronic transport in mesoscopic systems: For instance, the wave-packet time evolution [15, 16], the kernel polynomial expansion [17, 18], and the continued fraction expansion [19], to name a few. Recently, an alternative method to compute transport of ballistic graphene junctions, particularly effective when strong magnetic fields are presented, was introduced [20]. However, for most practitioners, the RGF remains the best method for tackling large-scale but finite-size problems where quantum coherence and disorder are present simultaneously.

This paper does not attempt to be a comprehensive review of the recursive method, but rather a self-contained description that gives to interested readers, yet unfamiliar with the RGF method, all the basic material necessary to implement a calculation on their own. For that purpose, we briefly present some standard material covered in textbooks [21, [22], discuss some more advanced issues which are found scattered in the literature, and present original developments tailor-made for graphene.

This paper is organized as follows. We begin by quickly reviewing some fundamental relations of electronic transport theory and by providing the essential formulation of the method. In Sec. 3 we present the recursive Green's function method. The method requires as input the surface Green's functions of the electronic leads, taken at the lead-device interface. In Sec. 4 we describe how to compute the surface Green's function of semi-infinite lattices that play the role of leads. Next, we present an efficient discretization scheme to implement the recursive method for graphene sheets and nanoribbons. In Sec. 6 we show how to evaluate quantities such as the local density of states and local current densities. Very often, one is interested in cases where the coherence length $\ell_{\phi}$ is comparable to the system size $L$. For such situations, it is possible to account for dephasing using the phenomenological voltage probe model, as described in Sec.7. In the context of graphene, the main application of the RGF method is the study of disorder effects in electronic transport. We discuss the main kinds of disorder and show how to account for them in Sec. 8. We conclude by presenting a number of numerical results that illustrate the method in Sec. 9

\section{Elements of Linear Mesoscopic Transport}

In this Section we review the key elements necessary to implement the recursive Green's function method for two- dimensional systems. The linear dc conductance is computed using the exact single-particle retarded Green's function that connects the source and drain leads, in conjunction with either the Landauer [23] or the Caroli formula [24].

For a two-probe setup, as illustrated by Fig. 1, the zerotemperature linear conductance is given by the Landauer formula

$\mathscr{G}=\frac{2 e^{2}}{h} \operatorname{Tr}_{c}\left[t^{\dagger} t\right]$

Here, $t\left(t^{\prime}\right)$ is the transmission matrix across the system from left to right (right to left) and $r\left(r^{\prime}\right)$ is the reflection matrix at the left-hand (right-hand) side. The factor of 2 stands for spin degeneracy and the trace is taken over the propagating modes at the left and right leads. The transmission matrix can be obtained from the $S$ matrix,

$S=\left(\begin{array}{cc}r & t^{\prime} \\ t & r^{\prime}\end{array}\right)$

which is given by [25, 26]

$$
\begin{aligned}
S_{a b}(E)=- & \delta_{a b}+i \hbar \sqrt{v_{a} v_{b}} \\
& \times \int d y_{q} \int d y_{p} \chi_{a}^{*}\left(y_{q}\right) G^{r}\left(y_{q}, y_{p} ; E\right) \chi_{b}\left(y_{p}\right),
\end{aligned}
$$

where $v_{c}$ and $\chi_{c}\left(y_{p}\right)$ are, respectively, the longitudinal propagation velocity and its transverse wave function in the propagating channel $c$ of lead $p$ (either on the left-hand or righthand side). The integrations run over the contact regions at the right and left terminations of the graphene sheet (see Fig. 11. The key element in Eq. (3) is $G^{r}\left(y_{q}, y_{p} ; E\right)$, the retarded Green's function corresponding to an electron with energy $E$ propagating from positions $y_{p}$ to $y_{q}$.

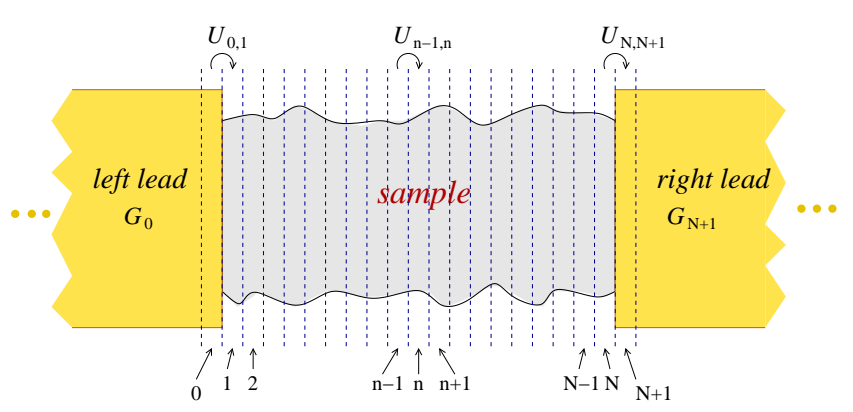

Fig. 1 Typical two-probe scheme used in the numerical calculations: The sample is described by $G\left(y_{q}, y_{p} ; E\right)$. The perfect leads can be accounted for by either propagating mode wave functions $\chi_{c}$ and their density of states $\rho_{c}$ (Landauer formula) or by level widths $\Gamma$ (Caroli formula).

The recursive Green's function method reviewed in this paper is an efficient tool to compute the scattering properties of noninteracting electrons described by a tight-binding 
Hamiltonian of the form

$H=-\sum_{i \neq j}\left(t_{i j}|i\rangle\langle j|+\right.$ H.c $)+\sum_{i} V_{i}|i\rangle\langle i|$.

In graphene, the hopping terms $t_{i j}$ typically connect only nearest neighbor sites $i$ and $j$ of a honeycomb lattice (singleorbital approximation), although it is straightforward to include next-to-nearest hopping terms as well, if required. The model can account for an external magnetic field by a suitable modification of the hopping terms, as shown in Appendix B. Here, $V_{i}$ stands for a local potential due to gating or disorder.

For simplicity, here we consider only tight-binding models with orthogonal orbitals and nearest-neighbor hopping terms. The computational cost of the RGF method scales as $N \times M^{3}$, where $N$ is the number of slices and $M$ is the typical number of sites in a given slice, see Fig. 11 The RGF implementation scheme presented in this paper is particularly recommended when the system's translational invariance is broken by disorder and/or an irregular geometry. For systems with translational invariance at the transverse direction, it can be advantageous to work in the $k$-space and use alternative hybrid RGF implementations (such as in Ref. [27]) or other methods, e.g. Ref. [20].

In the tight-binding basis, Eq. (3) reads

$S_{a b}(E)=-\delta_{a b}+i \hbar \frac{\sqrt{v_{a} v_{b}}}{a_{0}} \sum_{i \in p} \sum_{j \in q} \chi_{a}^{*}(i) G_{q p}^{r}(i, j) \chi_{b}(j)$,

where the sums run over the sites at the contacts $p$ and $q$ where the propagating channels $a$ and $b$ are defined, respectively. Notice that in two spatial dimensions

$$
\chi_{a}\left(y_{p}\right) \rightarrow \frac{1}{\sqrt{a_{0}}} \chi_{a}(i)=\frac{1}{\sqrt{a_{0}}}\left\langle i \mid \chi_{a}\right\rangle
$$

and

$$
G^{r}\left(y_{q}, y_{p}\right) \rightarrow \frac{1}{a_{0}^{2}} G_{q p}^{r}(j, i),
$$

where $a_{0}$ is the lattice constant. Let us consider the case where the leads are modeled by semi-infinite square lattices. One can then introduce the level widths [21]

$\Gamma_{p}\left(i, i^{\prime}\right)=\sum_{a} \chi_{a}(i) \frac{\hbar v_{a}}{a_{0}} \chi_{a}\left(i^{\prime}\right)$.

It is straightforward to show that, in this case,

$$
\operatorname{Tr}_{c}\left[t t^{\dagger}\right]=\sum_{\substack{i, i^{\prime} \in L \\ j, j^{\prime} \in R}} \Gamma_{L}\left(i, i^{\prime}\right) G_{L R}^{r}\left(i^{\prime}, j\right) \Gamma_{R}\left(j, j^{\prime}\right) G_{R L}^{a}\left(j^{\prime}, i\right)=\mathscr{T}
$$

where

$$
\mathscr{T} \equiv \operatorname{Tr}_{s}\left[\Gamma_{L} G_{L R}^{r} \Gamma_{R} G_{R L}^{a}\right]
$$

Here, the subscript in the trace indicates whether the sums run over channels $(c)$ or sites $(s)$. Depending on the author, the expression on the r.h.s. of Eq. (9) is called either Caroli [24] or Meir-Wingreen [28] conductance formula.

This demonstration of the equivalence between the Landauer and Caroli formulas relies on the Fisher and Lee $S$ matrix and on an expression for $\Gamma_{p}$ which is only suitable for a square lattice. This derivation is simple and to some extend non rigorous but captures the essential elements that will be discussed in what follows, namely, the Green's functions $G_{R L}^{(r, a)}$ and the decay width matrices $\Gamma_{R, L}$. There are several ways to show that (9) holds in general in the linear response regime, see e.g. Ref. [29].

When the full $S$ matrix is known, it is possible to obtain the global density of states through the Wigner time delay [30], namely,

$\rho(E)=-\frac{i}{2 \pi} \operatorname{Tr}_{c}\left(S^{\dagger} \frac{\partial S}{\partial E}\right)$

where the derivative of $S$ with respect to the energy can be done numerically. The computation of (11) is significantly less expensive than evaluating $\rho(E)$ through the standard expression, namely,

$\rho(E)=-\frac{1}{\pi} \operatorname{Im}\left[\operatorname{Tr}_{s^{\prime}} G^{r}(E)\right]$,

but it requires the knowledge of the explicit form of the lead wave functions $\chi_{a}(i)$. Note that, in Eq. (12), the trace is taken over all sites of the graphene sample.

The Fano factor is another quantity of interest [30]. It can be evaluated through the expression

$F=1-\frac{\operatorname{Tr}_{c}\left[t^{\dagger} t t^{\dagger} t\right]}{\operatorname{Tr}_{c}\left[t^{\dagger} t\right]}$

Notice that one can define left-to-right and right-to-left Fano factors, as in the case for the conductance, by switching the matrix $t$ with $t^{\prime}$. The Fano factor can be computed without an explicit knowledge of the wave functions $\chi_{a}(i)$ by noticing that Eq. (13) can be recast as

$F=1-\frac{\operatorname{Tr}_{s}\left[\Gamma_{L} G_{L R}^{r} \Gamma_{R} G_{R L}^{a} \Gamma_{L} G_{L R}^{r} \Gamma_{R} G_{R L}^{a}\right]}{\mathscr{T}}$.

Let us present the same basic expression in a more suitable form for the recursive calculations. We begin by writing the Caroli formula for the transmission probability at a given energy $E$ using the slice indexing,

$\mathscr{T}=\operatorname{Tr}_{s}\left[\Gamma_{L} G_{0, N+1}^{r}(E) \Gamma_{R} G_{N+1,0}^{a}(E)\right]$,

where $G^{r, a}$ are the retarded and advanced Green's functions across the system (see Fig. 2 for a definition of the subscripts 
in terms of slice numbers). These Green's functions are matrices whose rank is defined by the number of sites in the slices 11 The level width matrices are given by the expression

$\Gamma_{L, R}=i\left[\Sigma_{L, R}^{r}(E)-\Sigma_{L, R}^{r \dagger}(E)\right]$,

where the retarded surface self-energies of the leads read

$\Sigma_{L}^{r}(E)=u_{L} g_{L}^{r}(E) u_{L}^{\dagger} \quad$ and $\quad \Sigma_{R}^{r}(E)=u_{R}^{\dagger} g_{R}^{r}(E) u_{R}$.

Notice that the retarded Green's functions $g_{L}^{r}$ and $g_{R}^{r}$ are defined at the surface of the left and right leads, respectively, when the leads are decoupled from the system. They obey the self-consistent equations

$\left[E+i 0^{+}-h_{L}-\Sigma_{L}^{r}(E)\right] g_{L}^{r}(E)=I \quad$ and

$\left[E+i 0^{+}-h_{R}-\Sigma_{R}^{r}(E)\right] g_{R}^{r}(E)=I$,

where $h_{L}$ and $h_{R}$ are the Hamiltonians of isolated, individual slices in the left and right leads, respectively. The connection matrices $u_{L}$ and $u_{R}$ are defined to run from left-toright and are assumed uniform inside the leads. If the leads are identical, then $g_{R}^{r}=g_{L}^{r}, h_{L}=h_{R}, u_{R}=u_{L}^{\dagger}, \Sigma_{R}^{r}(E)=$ $\Sigma_{L}^{r}(E)$, and $\Gamma_{R}=\Gamma_{L}$. Notice that, in general, the coupling matrices are Hermitian, $\left(\Gamma_{R, L}\right)^{\dagger}=\Gamma_{R, L}$, while $\left(G^{r}\right)^{\dagger}=G^{a}$. These two properties guarantee that the transmission probability computed with Eq. (15) is always real. Moreover, since these coupling matrices are also positive by their definition in Eq. 16] [notice that the imaginary part of the retarded self-energy is negative if we adopt Eq. (18)], one can show that the transmission probability is positive, as it should be.

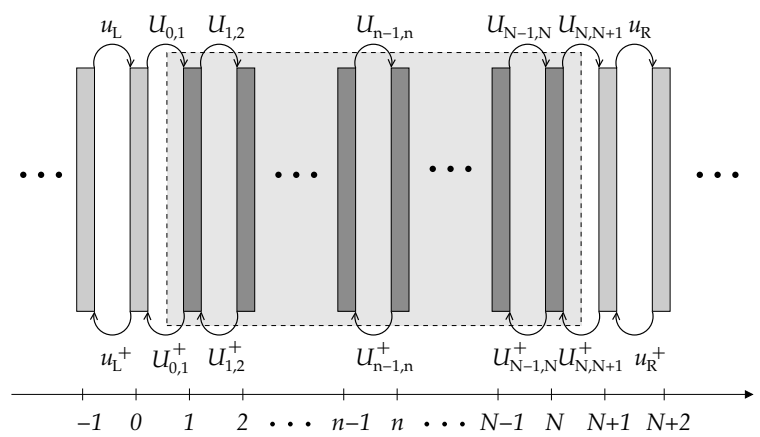

Fig. 2 Slicing scheme. The central rectangle containing the dark strips (slices) represents the bulk of the sample

\section{Recursive Green's Functions}

We now present the recursive Green's function method, a very efficient way to compute the Green's functions that appear in Eq. (15). We begin by introducing two equivalent

\footnotetext{
1 The number of sites per slice does not need to be equal for all slices.
}

Dyson formulas for an exact Green's function (for a derivation of these formulas, see Refs. [21,31]),

$G=G^{(0)}+G^{(0)} V G$,

$G=G^{(0)}+G V G^{(0)}$,

where $G^{(0)}$ represents the "unperturbed" Green's function and $V$ the perturbation. We use these expressions to obtain recursive relations for the exact Green's function of a quasione-dimensional system coupled to leads. The basic idea is to break up the system into independent parts (leads and slices) and associate to these parts "unperturbed" Green's functions $G^{(0)}$. The hoping matrix elements connecting those parts are then selectively built into the perturbation $V$. By choosing the connecting matrix elements and applying Eqs. (19) and (20) judiciously, we can build the full Green's function $G$ slice by slice.

Our presentation is specialized to the case of two-probe conductance, see Fig. 1. It is necessary to derive several intermediate recurrence formulas before obtaining expressions for the exact Green's function. We first run the recurrence from left to right, generating a family of Green's functions $G^{L}$. Thus, at every step, Eq. 190 is employed using a different choice for $G^{(0)}$ and $V$. We repeat the procedure from right to left, generating another set of functions $G^{R}$. Finally, we join these two families to obtain the exact $G$ for the whole system. In this way, all parts and connecting matrix elements are used (and never double counted).

The system is broken into $N$ thin slices, each one carrying a maximum of $M$ sites or cells, as show in Fig. 2. The slices with numbers lower than 1 or larger than $N$ represent the left and right leads, respectively. The corresponding retarded surface Green's functions (when the leads are decoupled from the system) are denoted by $g_{L}(E)$ and $g_{R}(E)$, as noted earlier. These Green's functions are computed separately and before the recurrence procedure (see $\mathrm{Sec}$. 4). The retarded Green's function of the isolated $n$th slice in the system, $g_{n}(E)=\left(E-h_{n}+i 0^{+}\right)^{-1}$, does not need to be individually evaluated before the recursive calculations. Here, $h_{n}$ denotes the Hamiltonian of the isolated $n$th slice.

Neighboring slices within the sample are connected to each other through the matrices $U_{n-1, n}$ (left to right) and $\left[U_{n-1, n}\right]^{\dagger} \equiv U_{n, n-1}$ (right to left), with $n=1, \ldots, N$. The first and last slices in the system are connected to their nearest neighboring slices in the leads through the coupling matrices $U_{0,1}$ and $U_{N, N+1}$. The matrix elements of these matrices are the tight-binding hopping amplitudes connecting sites at different slices.

Here, we assumed that the matrices $U$ only connect nearest-neighbor slices. For tight-binding models that include next-nearest hopping terms, one can still use this algorithm by doubling the "width" of the unit slices, which slows down the computation by a factor $2^{3}$. It also is possible to deal with 
next-nearest hopping terms and incur in a smaller slowdown factor by properly modifying the recursive method [32].

We use subscripts to denote longitudinal spatial indices (except for $g_{L}, g_{R}$, and $g_{n}$ ). Thus, $G_{n, m}(E)$ is the matrix Green's function connecting the $n$ and $m$ slices. Sites indices are shown as a pair of variables: $G_{n, m}\left(j, j^{\prime}\right)$ denotes the Green's function connecting site $j$ in the $n$th slice to site $j^{\prime}$ in the $m$ th slice. Hereafter, we will drop the energy variable $E$ (since scattering is assumed elastic, $E$ is conserved throughout the system).

\subsection{Connection to leads}

For the two-terminal setup we address here, the sample (central region) is coupled to a left lead $L$ and to a right lead $R$. In the following we show how to built the Green's function that describes this coupling.

We begin finding the Green's function $G^{L}$. We recall that the rightmost slice of the left lead if denoted by 0 . Our goal is to obtain $G_{0, n}^{L}$ and $G_{n, n}^{L}$ in order to describe electron propagation in the sample when the left lead is taken into account. The reason will become clear when we reach Sec. 3.4

The first step is to incorporate the $n=1$ slice to the left contact Green's function $g_{L}$. This kind of operation is repeated throughout the method and therefore we present it in detail. For this purpose, we introduce the kets $|0\rangle$ and $|1\rangle$ which represent the states where electrons are found in slices $n=0$ and $n=1$, respectively. The "unperturbed" Green's function in this case is $G^{(0)}=|0\rangle g_{L}\langle 0|+| 1\rangle g_{1}\langle 1|$ while $V=$ $|0\rangle U_{0,1}\langle 1|+| 1\rangle U_{1,0}\langle 0|$ is the perturbation that connects the $n=1$ slice to the left lead. Then, using Eq. 19], we obtain

$$
\begin{aligned}
\left\langle 1\left|G^{L}\right| 1\right\rangle & =\left\langle 1\left|G^{(0)}\right| 1\right\rangle+\sum_{m, m^{\prime}}\left\langle 1\left|G^{(0)}\right| m\right\rangle\left\langle m|V| m^{\prime}\right\rangle\left\langle m^{\prime}\left|G^{L}\right| 1\right\rangle \\
& =\left\langle 1\left|G^{(0)}\right| 1\right\rangle+\left\langle 1\left|G^{(0)}\right| 1\right\rangle\langle 1|V| 0\rangle\left\langle 0\left|G^{L}\right| 1\right\rangle
\end{aligned}
$$

and

$$
\begin{aligned}
\left\langle 0\left|G^{L}\right| 1\right\rangle & =\left\langle 0\left|G^{(0)}\right| 1\right\rangle+\sum_{m, m^{\prime}}\left\langle 0\left|G^{0}\right| m\right\rangle\left\langle m|V| m^{\prime}\right\rangle\left\langle m\left|G^{L}\right| 1\right\rangle \\
& =\left\langle 0\left|G^{0}\right| 0\right\rangle\langle 0|V| 1\rangle\left\langle 1\left|G^{L}\right| 1\right\rangle .
\end{aligned}
$$

Adopting the more compact notation $\left\langle n\left|G^{L}\right| m\right\rangle=G_{n, m}^{L}$, we drop the bras and kets and can rewrite these equation as

$G_{1,1}^{L}=g_{1}+g_{1} U_{1,0} G_{0,1}^{L}$

and

$G_{0,1}^{L}=g_{L} U_{0,1} G_{1,1}^{L}$.

Therefore,

$G_{1,1}^{L}=\left(I-g_{1} U_{1,0} g_{L} U_{0,1}\right)^{-1} g_{1}$.

Now, since $g_{1}=\left(E-h_{1}\right)^{-1}$, we can write

$G_{1,1}^{L}=\left(E-h_{1}-U_{1,0} g_{L} U_{0,1}\right)^{-1}$.
Notice that this Green's function takes into account the coupling of the first slice with the left lead, but has no information about the rest of the system or the right lead.

It is important to remark that we neglected the infinitesimal imaginary part in $g_{1}$ because we assumed that the "selfenergy" term in Eq. 26) brings its own finite imaginary part.

We proceed analogously in order to connect the last slice to the right lead. Choosing $G^{(0)}=g_{R}+g_{N}$ and $V=U_{N, N+1}$, we have

$G_{N, N}^{R}=g_{N}+g_{N} U_{N, N+1} G_{N+1, N}^{R}$

and

$G_{N+1, N}^{R}=g_{R} U_{N+1, N} G_{N, N}^{R}$.

(Note that the slice indices for the right Green's functions run opposite to those in the left Green's functions.) Therefore,

$G_{N, N}^{R}=\left(I-g_{N} U_{N, N+1} g_{R} U_{N+1, N}\right)^{-1} g_{N}$

Again, since $g_{N}=\left(E-h_{N}\right)^{-1}$, we can write

$G_{N, N}^{R}=\left(E-h_{N}-U_{N, N+1} g_{R} U_{N+1, N}\right)^{-1}$.

The Green's function $G_{1,1}^{L}$ (or $G_{N, N}^{R}$ ) describes all singleelectron processes that begin and end that on the $n=1$ (or $n=N$ ) slice, taking into account all possible number of incursions in and out of the left (or right) lead. It does not yet take into account incursions into the bulk of the system.

\subsection{Left Green's functions}

With $G_{1,1}^{L}$ in hand, we can evaluate the next successive $N-1$ left Green's functions by using a recurrence formula analogous to Eq. (26). To derive such formula, we choose $G^{(0)}=$ $G_{n-1, n-1}^{L}$ and $V=U_{n-1, n}+U_{n, n-1}$. Applying Eq. (19), we write

$G_{n, n}^{L}=\left(I-g_{n} U_{n, n-1} G_{n-1, n-1}^{L} U_{n-1, n}\right)^{-1} g_{n}$,

with $n=2, \ldots, N$. Using $g_{n}=\left(E-h_{n}\right)^{-1}$, we obtain

$G_{n, n}^{L}=\left(E-h_{n}-U_{n, n-1} G_{n-1, n-1}^{L} U_{n-1, n}\right)^{-1}$.

This formula is accompanied by another one, which connects the left-most slice (the surface slice of the left lead) with the $n$th one,

$G_{0, n}^{L}=G_{0, n-1}^{L} U_{n-1, n} G_{n, n}^{L}$.

Note that $N$ inversions are necessary to arrive at the $N$ th slice. Each inversion requires $O\left(M^{3}\right)$ operations. Thus, the complexity of the calculation scales as $N \times M^{3}$. 


\subsection{Right Green's functions}

Similarly to left case, for the right Green's functions, using Eq. (30) and starting from the $N$ th slice, we find that

$G_{n, n}^{R}=\left(I-g_{n} U_{n, n+1} G_{n+1, n+1}^{R} U_{n+1, n}\right)^{-1} g_{n}$,

with $n=N-1, \ldots, 1$. Substituting $g_{n}=\left(E-h_{n}\right)^{-1}$, we obtain

$G_{n, n}^{R}=\left(E-h_{n}-U_{n, n+1} G_{n+1, n+1}^{R} U_{n+1, n}\right)^{-1}$.

Also,

$G_{N+1, n}^{R}=G_{N+1, n+1}^{R} U_{n+1, n} G_{n, n}^{R}$.

Again, $N$ additional inversions have to be performed in order to arrive at slice the first slice $(n=1)$, with an overall computation cost $O\left(N \times M^{3}\right)$.

\subsection{Full Green's functions}

Suppose one arrives at the $n$ slice by either a left or right sweep $(1<n<N)$. To obtain the exact full Green's function of the system we use again Eq. (19) assuming $G^{(0)}=g_{n}+$ $G_{n-1, n-1}^{L}+G_{n+1, n+1}^{R}$, with $V=U_{n-1, n}+U_{n, n-1}+U_{n, n+1}+$ $U_{n+1, n}$. As a result, we find

$G_{n, n}=g_{n}+g_{n}\left(U_{n, n-1} G_{n-1, n}+U_{n, n+1} G_{n+1, n}\right)$,

$G_{n-1, n}=G_{n-1, n-1}^{L} U_{n-1, n} G_{n, n}$,

and

$G_{n+1, n}=G_{n+1, n+1}^{R} U_{n+1, n} G_{n, n}$.

Thus,

$$
\begin{aligned}
G_{n, n}=\left[I-g_{n}\left(U_{n, n-1} G_{n-1, n-1}^{L} U_{n-1, n}\right.\right. & \\
& \left.\left.+U_{n, n+1} G_{n+1, n+1}^{R} U_{n+1, n}\right)\right]^{-1} g_{n},
\end{aligned}
$$

and since $g_{n}=\left(E-h_{n}\right)^{-1}$, we obtain

$$
\begin{aligned}
G_{n, n}=\left(E-h_{n}\right. & -U_{n, n-1} G_{n-1, n-1}^{L} U_{n-1, n} \\
& \left.-U_{n, n+1} G_{n+1, n+1}^{R} U_{n+1, n}\right)^{-1},
\end{aligned}
$$

together with

$G_{0, n}=G_{0, n-1}^{L} U_{n-1, n} G_{n, n}$

and

$G_{N+1, n}=G_{N+1, n+1}^{R} U_{n+1, n} G_{n, n}$.

Note that in order to compute $G_{n, n}$ and $G_{N+1, n}$, we need to keep track of $G_{n, n}^{L}$ and $G_{n, n}^{R}$ [obtained recursively from
Eqs. (32) and (35), respectively], as well as $G_{0, n}^{L}$ and $G_{N+1, n}^{R}$ [which follow from Eqs. (33) and (36), respectively]. In order to obtain $G_{n-1, n}$ and $G_{n, n+1}$, we can apply Dyson's equation again to a situation where only the $n$th slice is decoupled, yielding

$G_{n, n+1}=G_{n, n} U_{n, n+1} G_{n+1, n+1}^{R}$,

while

$G_{n-1, n}=G_{n-1, n-1}^{L} U_{n-1, n} G_{n, n}$.

These equations are useful for computing the local current distribution (Sec. 6).

We note that when computing the exact Green's in Eqs. (41), 42), (43), 44), and 45) we have selectively used each matrix $U_{n, n^{\prime}}$ only once. Similarly, at each step, an isolated slice Hamiltonian $h_{n}$ was used and never repeated. Thus, at the end of the calculation of the full Green's function, all hoping amplitudes and local potentials of the underlying tight-binding model have been used and only once.

An alternative way to compute full Green's functions, which is quite useful if only transmission and reflection matrices are required, is to close the left (or right) sweep with a connection to the right (left) lead:

1. For the left sweep, we use Eq. (42) to write

$G_{0, N+1}=G_{0, N}^{L} U_{N, N+1} G_{N+1, N+1}$,

which is complemented by

$G_{N+1, N+1}=\left(g_{R}^{-1}-U_{N+1, N} G_{N, N}^{L} U_{N, N+1}\right)^{-1}$

obtained from Eq. (41).

2. For the right sweep, we use instead Eqs. (43) and (42) to obtain

$G_{N+1,0}=G_{N+1,1}^{R} U_{1,0} G_{0,0}$

and

$G_{0,0}=\left(g_{L}^{-1}-U_{0,1} G_{1,1}^{R} U_{1,0}\right)^{-1}$,

respectively.

As we will see below, Eqs. (46) and 49) and can be used to compute the left-to-right transmission and left reflection matrices, respectively, while Eqs. (48) and (47) yield the right-to-left transmission and the right reflection matrices. For systems with inversion symmetry, we expect $G_{00}=$ $G_{N+1, N+1}$ and $G_{0, N+1}=G_{N+1,0}$ and therefore only one sweep (left or right) is necessary for the evaluation of the whole scattering matrix.

For symmetric leads and in the absence of an external magnetic field (i.e., time-reversal symmetric systems),

$\left[G_{0, N+1}^{r}\right]^{\dagger}=G_{N+1,0}^{a}$ 
and only one sweep is necessary. When such conditions are not met, one needs both sweeps, namely, from left-to-right and from right-to-left, in order to assemble the scattering matrix. Moreover, any local observable (such as the local density of states or the local current flux), requires $G_{0, N+1}$ as well as $G_{n, n}$ for all $n=1, \ldots, N$.

\subsection{Input Green's functions}

The recurrence relations shown above rely on some input information. One needs to define the Green's functions of the leads $\left(g_{L}\right.$ and $\left.g_{R}\right)$, the Hamiltonian of the isolated slices $\left(h_{n}\right.$, $n=1, \ldots, N$ ), and the hopping between slices (the $U$ matrices) before starting the calculation of the sample's Green's function.

Since both $g_{L}$ and $g_{R}$ are the input Green's functions, it is crucial that they have finite imaginary parts. These will be dominant and, in practice, we can basically neglect the imaginary part when considering $g_{n}$ (even if $E$ happens to coincide with an eigenvalue of an isolated slice, the imaginary parts brought in by coupling to the leads makes the Green's function convergent). We will see next how to obtain contact Green's functions for leads modeled as semiinfinite lattices.

\section{Lead Green's Functions}

To satisfactorily model the leads, there are two main physical considerations to keep in mind: (a) the source and drain leads in typical graphene transport experiments are metallic and thus have a high density of states; (b) graphene-metal interfaces tend to form ohmic contacts. Thus, in the numerical simulations, one needs to eliminate or minimize the contact resistance associated to band structure mismatch at the contacts. To address (a), the chemical potential in the leads is customarily adjusted to maximize the density of states. To address (b), an appropriate lead lattice model, compatible with the sample lattice, is chosen to minimize back reflections. The leads are usually modeled either by square or honeycomb semi-infinite lattices [33]. In certain cases, a combination of square lattice contacts coupled to semi-infinite linear chains are shown to be advantageous to minimize the contact resistance [34].

The methods to compute the lead Green's functions can be divided into two categories, namely, the iterative recursive methods [35] and the eigenchannel decomposition or mode matching ones [11,36, 37, 38]. In the latter, the lead Green's function are built with the eigenchannels of the infinite (translation invariant) corresponding lattice. Except for few cases, such as the semi-infinite square lattice discussed below, the eigenmodes depend on the longitudinal wave num- ber $k$ and the $g_{L, R}$ cannot be written in closed analytical form.

In this Section we review the eigenchannel decomposition of $g_{L, R}$ for semi-infinite square lattices and discuss how to couple $g_{L, R}$ to a graphene device. Next, we present the decimation method for semi-infinite lattices [35]. Despite the claim that, in general, iterative methods are inferior in performance and accuracy than the eigendecomposition ones [36], the decimation method has the attractive properties of being very robust and straightforward, allowing for a very amenable implementation.

\subsection{Square lattice leads - analytical approach}

Let us model the contacts by semi-infinite tight-binding square lattices [33]. Let us also set the Fermi energy to $E=0$ [39. 33]. We can shift the energy band of the electronic states in the leads by varying a gate potential $V_{\text {lead }}$ in the leads 40 , 39,33]. At zero bias and in the absence of inelastic scattering, this sets the energy of the electrons propagating through the graphene sheet.

The electron wave function in the semi-infinite square lattice is extended along the $x$-direction and is quantized in the transverse direction. Let us consider hard-wall boundary conditions at the edges of the strip, namely, $j=0$ and $j=$ $M+1$. The transverse wave functions are given by

$\chi_{v}(j)=\sqrt{\frac{2}{M+1}} \sin \left(\frac{\pi v j}{M+1}\right)$,

where $v=1, \cdots, M$. Associated to each transverse mode there are two extended Bloch waves with longitudinal wave numbers $\pm k_{n}$, which are real for propagating modes and complex for evanescent modes.

For convenience, we assume [22] a hard-wall boundary condition at the left end of the strip (where it connects to the right Green's function of the strip), such that $\phi_{\mu}(N-1)=0$ :

$\phi_{\mu}(n)=\sqrt{\frac{2}{\pi}} \sin [\mu(n-N+1)]$,

where $\mu$ is a longitudinal quantum number. When contrasted with a continuum model, we can identify $\mu=k_{x}^{\mu} a_{x}$, where $k_{x}^{\mu}$ is the longitudinal wave vector. By choosing $\phi_{\mu}(N-1)=$ 0 we are not capable of treating the contribution of evanescent modes. This is usually not a problem in practice, unless measurements are done very close to the contacts (i.e., for very short systems).

The dispersion relation for this model is

$E_{v \mu}=V_{\text {lead }}-2 t_{x} \cos \mu-2 t_{y} \cos \left(\frac{\pi v}{M+1}\right)$.

The velocities of the propagating modes are

$v_{v}=\frac{a_{0}}{\hbar}\left(\frac{d E_{v \mu}}{d \mu}\right)=\frac{2 a_{0} t_{x}}{\hbar} \sin \mu$. 
By setting $E_{v \mu}=0$, one writes $\sin \mu=\sqrt{1-\cos ^{2} \mu}$ with $\cos \mu=V_{\text {lead }} / 2 t_{x}-\left(t_{y} / t_{x}\right) \cos [\pi v /(M+1)]$.

Let us now construct the leads Green's function as in Ref. [22]. The general expression

$g\left(n, j ; n^{\prime}, j^{\prime} ; E\right)=\int_{0}^{\pi} d \mu \sum_{v=1}^{M} \frac{\left[\phi_{\mu}(n) \chi_{v}(j)\right]^{*}\left[\phi_{\mu}\left(n^{\prime}\right) \chi_{v}\left(j^{\prime}\right)\right]}{E-E_{v \mu}+i 0^{+}}$

can be simplified since we are only interest in $n=n^{\prime}=N$ (surface) and $E=0$. It reads

$g_{L}\left(j, j^{\prime}\right)=\frac{2}{\pi} \sum_{v=1}^{M} \chi_{v}(j)^{*} \chi_{v}\left(j^{\prime}\right) \int_{0}^{\pi} d \mu \frac{\sin ^{2} \mu}{p+q \cos \mu}$,

where

$p \equiv-V_{\text {lead }}+2 t_{y} \cos \left(\frac{\pi v}{M+1}\right)+i 0^{+}$and $q \equiv 2 t_{x}$.

By integrating over $\mu$, one writes [22]

$G_{N}^{\mathrm{semi}}\left(j, j^{\prime}\right)=\sum_{v=1}^{M} \chi_{v}^{*}(j) \widetilde{G}^{\mathrm{semi}}(v) \chi_{v}\left(j^{\prime}\right)$

where

$\widetilde{G}^{\text {semi }}(v)=\frac{2 p}{q^{2}}\left[1-\sqrt{1-\left(\frac{q}{p}\right)^{2}}\right]$.

Equation (58) defines the unitary transformation that converts the Green's function $\widetilde{G}^{\text {semi }}(v)$ in the channel representation into $G_{N}^{\text {semi }}\left(j, j^{\prime}\right)$ in the site representation.

In Ref. [33], Schomerus argues that these expressions can be related to the parameter $\mu$ for graphene semi-infinite lattices in the case of armchair edge orientation. He shows that $\widetilde{G}^{\text {semi }}(\widetilde{v})=-\mu^{\operatorname{armchair}}\left(V_{\text {lead }}\right) / t_{x}^{2}$. This correspondence works well for armchair orientations because in that case propagating modes do not mix; it does not work for zigzag or other edge orientations. In Ref. [39] the authors speculate that transport properties in the presence of bulk disorder should not depend on the graphene orientation, which is numerically confirmed in Ref. [40]. Thus, in large-scale numerical simulations involving bulk disorder, it is worth taking advantage of the matching between square-lattice leads and graphene armchair leads.

\subsection{Eigenmode decomposition method - square lattice}

The following alternative approach to obtain the surface Green's function of a square-lattice lead is helpful since the same steps can be repeated for any other lattice and they form the basis of the eigendecomposition methods.
Since adding another slice to a semi-infinite lead should not alter its Green's function, we can write that, in the absence of any disorder or inhomogeneity,

$G_{1,1}^{L}=g_{L}$

when $U_{0,1}=-t_{x} I$, where $t_{x}>0$ is the horizontal hopping matrix element. Then, using Eq. 26, we obtain the selfconsistency condition

$g_{L}=\left(E-h_{1}-t_{x}^{2} g_{L}\right)^{-1}$.

Here, we assumed $U_{1,0}=t_{x}^{2} I$, appropriate for square lattices, where $I$ is the identity matrix. In order to solve Eq. 61 for $g_{L}$, we notice that since $h_{1}$ is Hermitian, it must be diagonalizable by a unitary transformation: $T^{\dagger} h_{1} T=\operatorname{diag}\left(\varepsilon_{v}\right)$, where $\operatorname{diag}\left(\varepsilon_{V}\right)$ is a diagonal matrix containing the eigenvalues of $h_{1}$. Then,

$\tilde{g}_{L}(v)=\left(E-\varepsilon_{v}-t_{x}^{2} \tilde{g}_{L}(v)\right)^{-1}$,

where $\operatorname{diag}\left(\tilde{g}_{L}(v)\right)=T^{\dagger} g_{L} T$ is the diagonal matrix containing the eigenvalues of $g_{L}$. Solving Eq. 62, we find

$\tilde{g}_{L}(v)=\frac{\left(E-\varepsilon_{v}\right)}{2 t_{x}^{2}} \pm \sqrt{\frac{\left(E-\varepsilon_{v}\right)^{2}}{4 t_{x}^{4}}-\frac{1}{t_{x}^{2}}}$.

Notice that for $\left|E-\varepsilon_{v}\right|<2 t_{x}$, this eigenvalue acquires a finite imaginary part. Since we are mainly interested in retarded Green's functions, we choose the negative sign and rewrite the equation as

$\tilde{g}_{L}^{r}(v)= \begin{cases}\frac{\left(E-\varepsilon_{v}\right)}{2 t_{x}^{2}}\left[1-\sqrt{1-\frac{4 t_{x}^{2}}{\left(E-\varepsilon_{v}\right)^{2}}}\right]+i 0^{+}, & \left|E-\varepsilon_{v}\right| \geq 2 t_{x}, \\ \frac{\left(E-\varepsilon_{v}\right)}{2 t_{x}^{2}}-i \sqrt{\frac{1}{t_{x}^{2}}-\frac{\left(E-\varepsilon_{v}\right)^{2}}{4 t_{x}^{4}}}, & \left|E-\varepsilon_{v}\right|<2 t_{x} .\end{cases}$

We need now to determine $T$ and $\left\{\varepsilon_{v}\right\}$. For a square lattice, this is trivial: $h_{1}$ describes a one-dimensional chain with $M$ sites and vertical hopping matrix elements $t_{y}>0$ (hard boundary conditions assumed). Then,

$T_{v j}=\sqrt{\frac{2}{M+1}} \sin \left(\frac{\pi v j}{M+1}\right)=\chi_{v}(j)$,

where $j=1, \ldots, M$ and $v=1, \ldots, M$, and

$\varepsilon_{v}=V_{\text {lead }}-2 t_{y} \cos \left(\frac{\pi v}{M+1}\right)$

resulting in

$g_{L}\left(j, j^{\prime}\right)=\sum_{v=1}^{M} \chi_{v}(j) \tilde{g}_{L}(v) \chi_{v}\left(j^{\prime}\right)$.

In Appendix $\mathrm{A}$, we show that Eq. 67) gives the expected steps in the linear conductance. 
Unfortunately, the above expressions do not directly apply to either zigzag or armchair leads, basically because $U_{0,1}$ is not proportional to the identity in these cases and it does not commute with the slice Hamiltonian. The solution for the general case is nicely presented in Ref. [38].

\subsection{Leads Green's function - decimation method}

We can use the decimation method of Ref. [35] to evaluate numerically the Green's function of leads with arbitrary (but translation invariant) lattice structures.

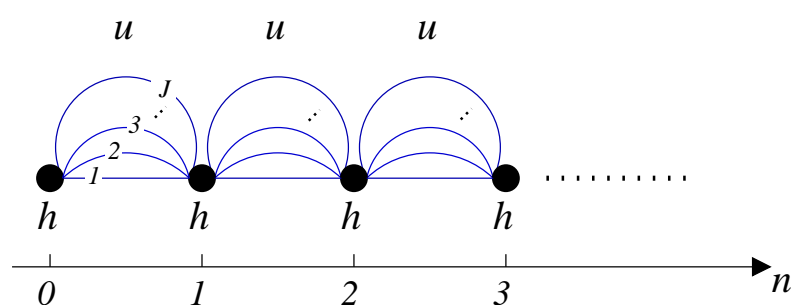

Fig. 3 Chain structure used in the decimation method.

The method works as follows. Suppose we want to solve $(E-H) G(E)=I$ for the operator $G(E)$, where the Hamiltonian operator $H$ is defined over a chain where each site has an arbitrary basis of dimension $J$ but only nearest-neighbor inter-site connections exist (see Fig. 3). We assume that all these connections are represented by operators $u$ (left-toright) and $u^{\dagger}$ (right-to-left). The local Hamiltonian is identical for all sites and denoted by $h$. Since, by definition, the Green's function obeys

$[(E-H) G(E)]_{n, m}=\delta_{n, m}$,

it follows that

$(E-h) G_{0,0}=I+u G_{1,0}$,

$(E-h) G_{1,0}=u G_{2,0}+u^{\dagger} G_{0,0}$,

$(E-h) G_{n, 0}=u G_{n+1,0}+u^{\dagger} G_{n-1,0}$,

with $n \geq 1$. Using Eq. (70), we find that

$G_{1,0}=(E-h)^{-1}\left(u G_{2,0}+u^{\dagger} G_{0,0}\right)$,

which can be combined with Eq. (69) to yield

$(E-h) G_{0,0}=I+u(E-h)^{-1}\left(u G_{2,0}+u^{\dagger} G_{0,0}\right)$,

which can be rewritten as

$\left[E-h-u(E-h)^{-1} u^{\dagger}\right] G_{0,0}=I+u(E-h)^{-1} u G_{2,0}$.

Notice that we can relate $G_{2,0}$ to $G_{0,0}$ without involving $G_{1,0}$.
The same trick can be employed for any value of $n$. From Eq. (71), we can write

$G_{n+1,0}=(E-h)^{-1}\left(u G_{n+2,0}+u^{\dagger} G_{n, 0}\right)$,

and

$G_{n-1,0}=(E-h)^{-1}\left(u^{\dagger} G_{n-2,0}+u G_{n, 0}\right)$.

These equations can be combined with Eq. (71) to yield

$(E-h) G_{n, 0}=u(E-h)^{-1}\left(u G_{n+2,0}+u^{\dagger} G_{n, 0}\right)+$ $u^{\dagger}(E-h)^{-1}\left(u G_{n, 0}+u^{\dagger} G_{n-2,0}\right)$

which can be rewritten as

$$
\begin{aligned}
& {\left[E-h-u(E-h)^{-1} u^{\dagger}-u^{\dagger}(E-h)^{-1} u\right] G_{n, 0}=} \\
& u(E-h)^{-1} u G_{n+2,0}+u^{\dagger}(E-h)^{-1} u^{\dagger} G_{n-2,0}
\end{aligned}
$$

Equations (74) and (78) generate a new recursion series involving only even sites:

$\left(E-\varepsilon_{1}^{s}\right) G_{0,0}=I+\alpha_{1} G_{2,0}$
$\left(E-\varepsilon_{1}\right) G_{2,0}=\alpha_{1} G_{4,0}+\beta_{1} G_{0,0}$

$\left(E-\varepsilon_{1}\right) G_{n, 0}=\alpha_{1} G_{n+2,0}+\beta_{1} G_{n-2,0}$

with

$\alpha_{1}=u(E-h)^{-1} u$

$\beta_{1}=u^{\dagger}(E-h)^{-1} u^{\dagger}$

$\varepsilon_{1}^{s}=h+u(E-h)^{-1} u^{\dagger}$

$\varepsilon_{1}=\varepsilon_{1}^{s}+u^{\dagger}(E-h)^{-1} u$.

Even though Eqs. (79) to (81) involve only even sites (i.e., multiples of $2^{1}$ ), they are identical in form to Eqs. (70) and (71). Therefore, we can repeat this procedure $k$ times until the recursion relations involve only sites that are multiple of $2^{k}$, namely,

$$
\begin{aligned}
\left(E-\varepsilon_{k}^{S}\right) G_{0,0} & =I+\alpha_{k} G_{2,0} \\
\left(E-\varepsilon_{k}\right) G_{2^{k}, 0} & =\alpha_{k} G_{2^{k} \cdot 2,0}+\beta_{k} G_{0,0} \\
& \vdots \\
\left(E-\varepsilon_{k}\right) G_{2^{k} \cdot n, 0} & =\alpha_{k} G_{2^{k} \cdot(n+1), 0}+\beta_{k} G_{2^{k} \cdot(n-1), 0}
\end{aligned}
$$

with $n \geq 1$ and

$$
\begin{aligned}
\alpha_{k} & =\alpha_{k-1}\left(E-\varepsilon_{k-1}\right)^{-1} \alpha_{k-1}, \\
\beta_{k} & =\beta_{k-1}\left(E-\varepsilon_{k-1}\right)^{-1} \beta_{k-1}, \\
\varepsilon_{k}^{s} & =\varepsilon_{k-1}+\alpha_{k-1}\left(E-\varepsilon_{k-1}\right)^{-1} \beta_{k-1}, \\
\varepsilon_{k} & =\varepsilon_{k}^{s}+\beta_{k-1}\left(E-\varepsilon_{k-1}\right)^{-1} \alpha_{k-1} .
\end{aligned}
$$

The decimation can stop when $\left\|\alpha_{k}\right\|$ and $\left\|\beta_{k}\right\|$ are sufficiently small, in which case we can approximate

$G_{0,0} \approx\left(E-\varepsilon_{k}^{s}\right)^{-1}$. 


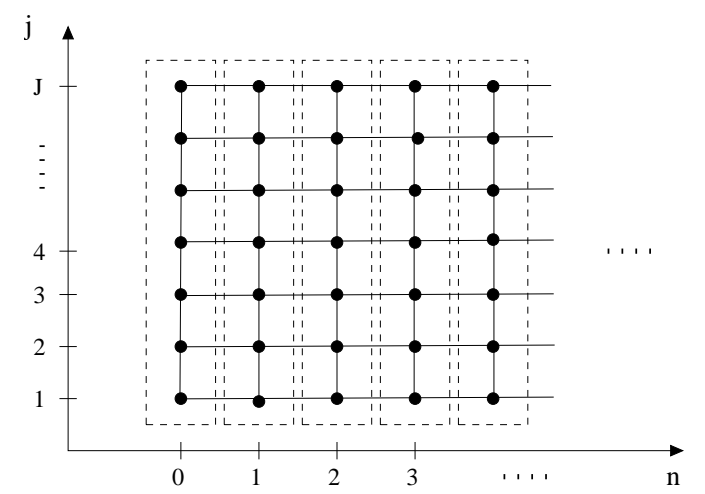

Fig. 4 Structure of the square lattice lead. The slice with $n=0$ corresponds to the "surface" which will be attached to the system. Slices for decimation are denoted by the boxes with dashed lir ${ }^{-}$

This provides the Green's function for the which can then be related to the lead Greer and $G_{N+1}^{R}$.

Note that one needs to add a small po: part to $E$, namely, $E \rightarrow E+i \eta$, in order to gi Green's functions. On the practical side, a helps to speed up the convergence but spo of $G_{0,0}^{r}$ at the order of $\eta / t$. Provided that $r$ an imaginary part to $E$ has little effect on 1 of graphene transport properties for $|E| / \eta$ a small energy interval around the charge 1 where $E=0$.

\subsubsection{Square lattice lead}

From Fig. 4 we see that each decimation sit a regular open vertical chain with $J$ lattice s

$h\left(j, j^{\prime}\right)=\sum_{\mu=1}^{J} \phi_{\mu}(j) \phi_{\mu}\left(j^{\prime}\right) E_{\mu}$,

and

$u\left(j, j^{\prime}\right)=-t \delta_{j, j^{\prime}}$.

where $t$ is the nearest-neighbor hopping amplitude, $j, j^{\prime}=$ $1, \ldots, J, \mu=1, \ldots, J$,

$$
\begin{aligned}
\phi_{\mu}(j) & =\sqrt{\frac{2}{J+1}} \sin \left(\frac{\pi \mu j}{J+1}\right), \quad \text { and } \\
E_{\mu} & =-2 t \cos \left(\frac{\pi \mu}{J+1}\right) .
\end{aligned}
$$

From Eqs. (94) and (95), we derive the relations

$\alpha_{1}\left(j, j^{\prime}\right)=t^{2}\left[(E-h)^{-1}\right]_{j, j^{\prime}}$,

$\varepsilon_{1}^{s}\left(j, j^{\prime}\right)=h\left(j, j^{\prime}\right)+\alpha_{1}\left(j, j^{\prime}\right)$, and

$\varepsilon_{1}^{s}\left(j, j^{\prime}\right)=h\left(j, j^{\prime}\right)+2 \alpha_{1}\left(j, j^{\prime}\right)$.

Figure 5 shows a comparison between the analytical and decimation results for the $G^{r}(E)$ of a square lattice of width $J=M=6$, projected onto the eigenchannel basis. Notice the excellent agreement.

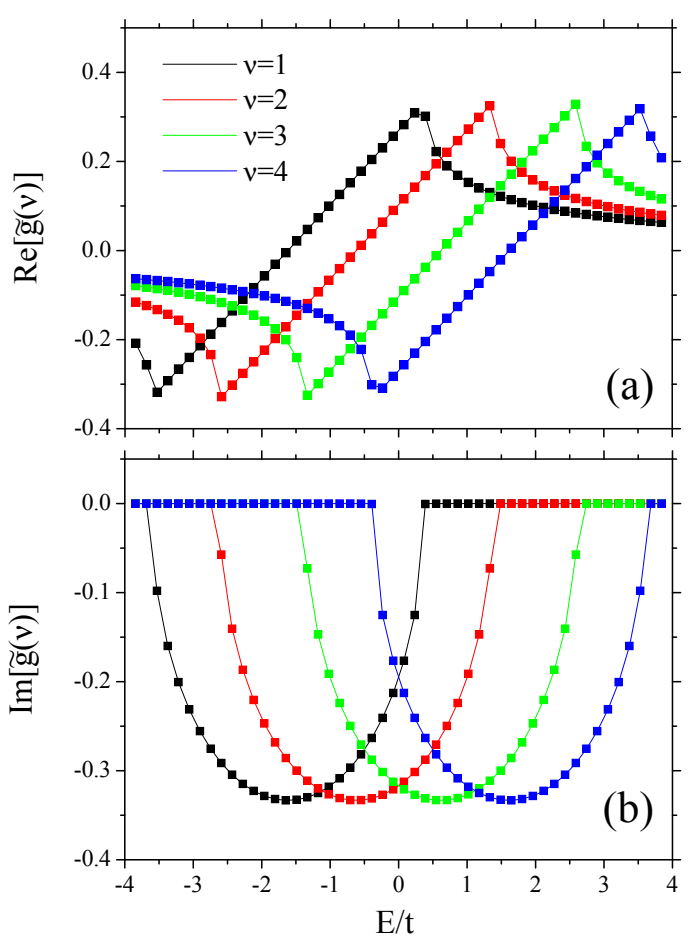

Fig. 5 Real (a) and imaginary part (b) of the Green function of a semiinfinite square lattice in the channel (diagonal) representation, $\widetilde{g}(\mu)$, as a function of energy. $J=4$. The solid lines represent the exact analytic result, whereas the squares were numerically obtained through the decimation method for $\eta=10^{-6}$ and $k=10$.

\subsubsection{Honeycomb lattice lead-armchair edges}

For the honeycomb lattice with armchair edges, the elementary slice contains $J$ stacked hexagons. Thus, $M=2 J+1$, where $M$ is the vertical number of atoms. The slice has the structure of a vertical ladder chain ( $2 M$ atoms). In this case there is no simple formula for the eigenstates of $h$. The ma- 


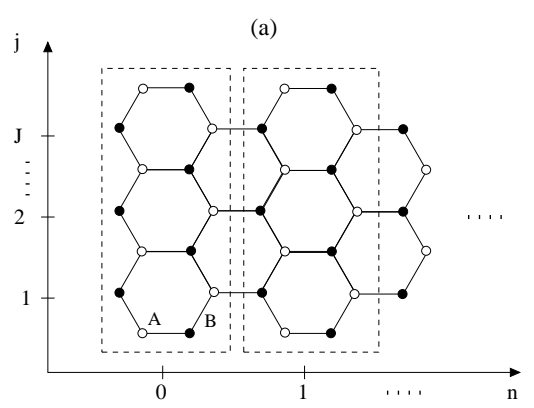

(b)

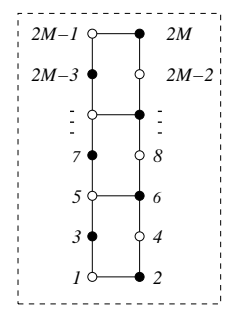

Fig. 6 (a) Structure of the honeycomb armchair lead. Note that the number of interconnect channels runs from 1 to $J$, while the number of vertical coordinate points in the slice is $M=2 J+1$. The slices for decimation are defined by the boxed regions. (b) Internal index structure of the elementary slice. (a)

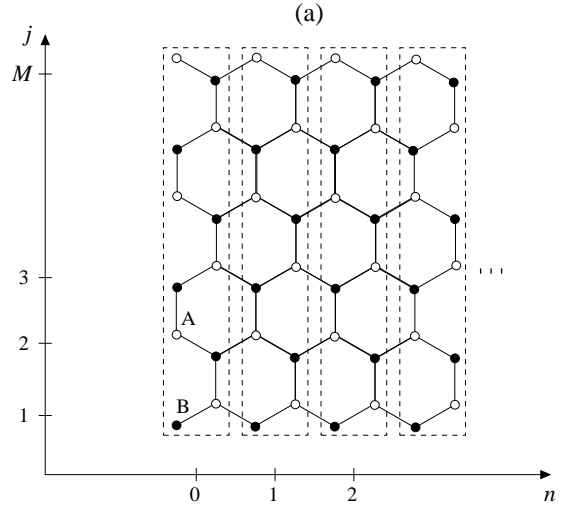

(b)

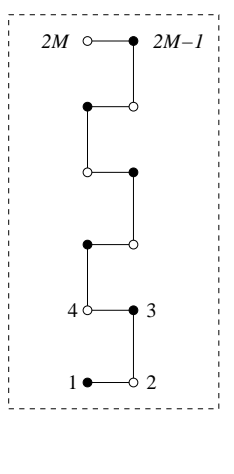

Fig. 7 (a) Structure of the honeycomb zigzag lead. Note that the number of interconnect channels runs from 1 to $J=M$, where $M$ is the number of vertical coordinate points in the graphene. The slices for decimation are defined by the boxed regions. (b) Internal index structure of the elementary slice.

trix $h$, which is $2 M \times 2 M$ dimensional, reads

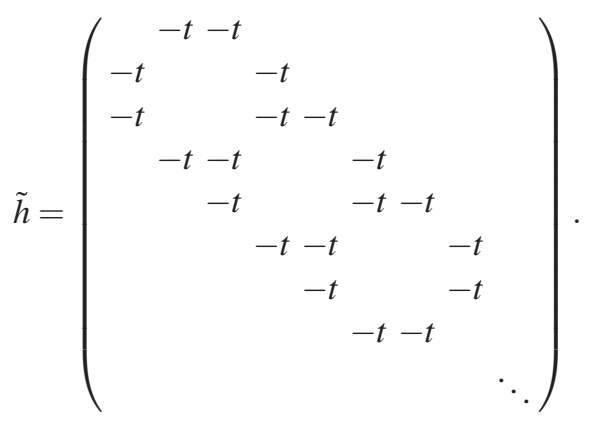

Let us call $\left\{\phi_{\mu}(m)\right\}$ its eigenvectors and $\left\{E_{\mu}\right\}$ its eigenvalues, with $\mu=1, \ldots, 2 M$. Then,

$h\left(j, j^{\prime}\right)=\sum_{\mu=1}^{2 M} \phi_{\mu}(4 j) \phi_{\mu}\left(4 j^{\prime}-1\right) E_{\mu}$,

with $j, j^{\prime}=1, \ldots, J$. Everything else is similar to Sec.4.3.1.

\subsubsection{Honeycomb lattice lead - zigzag edges}

For the zigzag graphene lead, there is a direct correspondence between slice channels and the vertical indices of atomic positions: $M=J$. The elementary slice is a open vertical chain of length $2 M$. Its eigenfunctions and eigenvalues are

$\phi_{\mu}(l)=\sqrt{\frac{2}{2 M+1}} \sin \left(\frac{\pi \mu l}{2 M+1}\right)$, and
$E_{\mu}=-2 t \cos \left(\frac{\pi \mu}{2 M+1}\right)$

with $l=1, \ldots, M$ and $\mu=1, \ldots, 2 M$. The slice matrix reads

$h\left(j, j^{\prime}\right)=\sum_{\mu=1}^{2 M} \phi_{\mu}(2 j-1) \phi_{\mu}\left(2 j^{\prime}\right) E_{\mu}$,

with $j, j^{\prime}=1, \ldots, M$. All other aspects are identical to Sec. 4.3 .1 .

\section{Device Green's Function}

The device Green's function $G_{i, j}$ can be used to describe nearly all the physics of transport and its calculation is where most of the computational time is spent. This is where any optimization of the computational method is most welcome, particularly in the study of disorder effects in the electronic transport when extensive disorder averaging is required. With the RGF method one can compute $G_{i, j}$ for a large variety of settings. For instance, the graphene sheet can have an arbitrary number of layers and different edge orientations, namely, zigzag, armchair, and chiral. Also, the tight-binding model can include next-nearest neighbor hopping terms in addition to the nearest neighbor ones. These elements have to be taken into account when choosing the slice unit cell employed by the recursive method. The description of disorder modeling is postponed to section 8 .

In this Section we present efficient slicing schemes for graphene monolayers with armchair and zigzag edges.

\subsection{Slicing armchair lattices}

For rectangular geometries, the bottleneck of the recursive method is the matrix inversion required for adding a new slice [see Eq. 32] ]. Thus, it is always important to try to minimize the number of sites in the slice. Having this in mind, there is a way to mount the armchair slices which reduces the number of sites per slice without introducing next-to-nearest neighbor hopping. It is based on the lattice deformation shown in Fig. 8 .

The relations between lattice size and graphene sheet dimensions for the efficient armchair slicing are

$\frac{L}{a_{0}}=\frac{\sqrt{3}}{2}\left(\frac{N}{2}-1\right)+\frac{\sqrt{3}}{6}$ and $\frac{W}{a_{0}}=M-1$. 


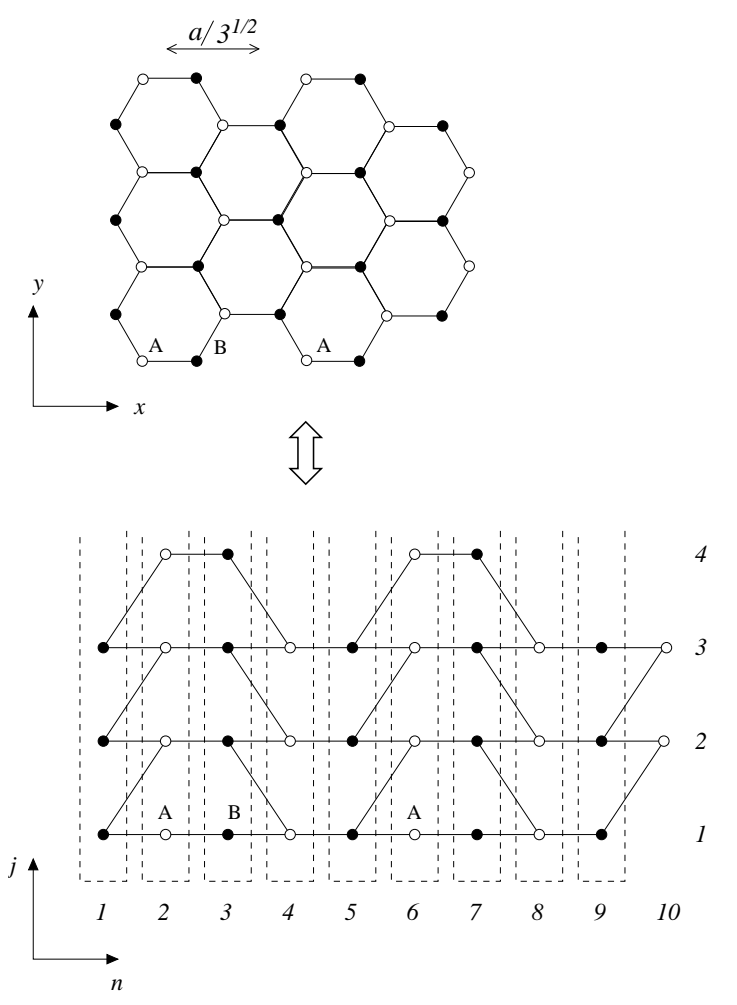

Fig. 8 Efficient way to slice an armchair graphene ribbon (the socalled "pine tree" configuration). Atoms from different sublattices are indicated by empty (A) and full (B) circles. $a_{0}$ is the lattice constant. The dashed lines indicate vertical slices.

\subsection{Slicing zigzag lattices}

The zigzag geometry is shown in Fig. 9 The real structure is shown on the left-hand side (honeycomb lattice). An equivalent square lattice with missing vertical bonds is also shown (the so-called "brick wall" configuration). For zigzag edge ribbons there is no efficient, alternative slicing that minimizes the number of sites per slice without creating a nextto-nearest neighbor connectivity.

By convention, we assume that the site at the left bottom corner of the lattice is of type A. In that way, slices with even and odd number of sites will alternate as we move horizontally. Two cases will need to be considered separately: $M$ odd and $M$ even. However, both cases share a common trend, namely, the presence of dimers and isolated sites (at the bottom and/or at the top of the slice). Therefore, finding the Green's function of an isolated slice is a very simple exercise.

The relations between lattice size and graphene sheet dimensions for the zigzag slicing are

$$
\frac{W}{a_{0}}=\frac{\sqrt{3}}{2}(M-1)+\frac{\sqrt{3}}{6} \text { and } \quad \frac{L}{a_{0}}=\frac{N-1}{2} .
$$
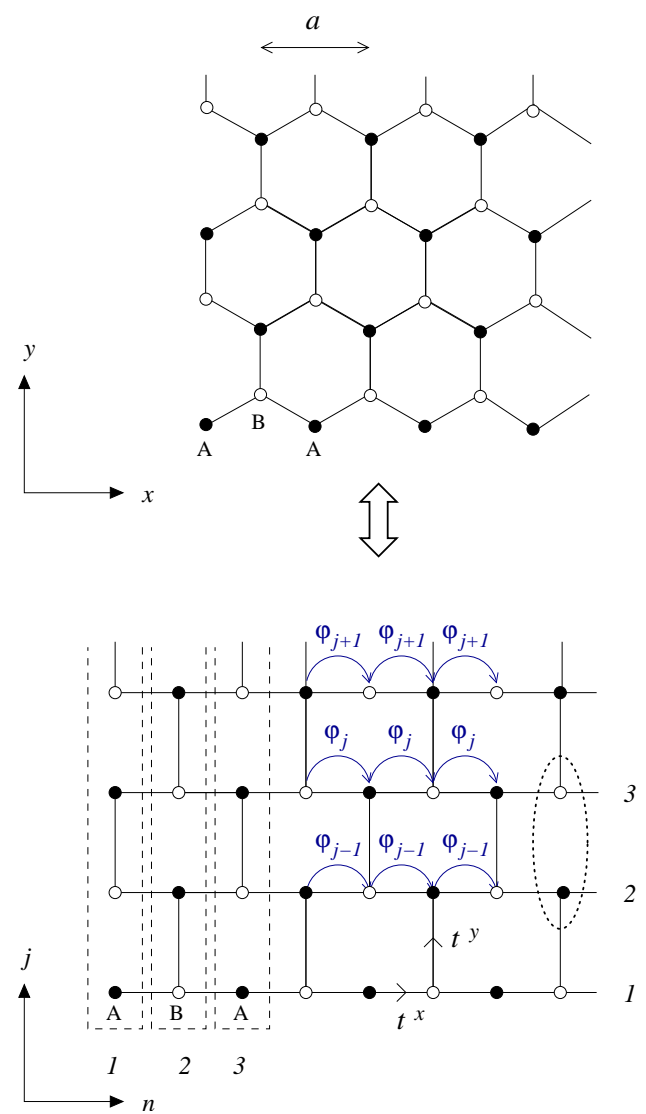

Fig. 9 Graphene strip with zigzag edges. Atoms from different sublattices are indicated by empty (A) and full (B) circles. $a_{0}$ is the lattice constant. The dashed lines indicate vertical slices and the dotted highlights a dimer. By convention, we set as $(1,1)$ the coordinates of an atom of type A placed at the left bottom corner. The Peierls phases (see appendix B of the "horizontal" hopping matrix elements are indicated.

\section{Evaluating Local Quantities}

In addition to the transmission $\mathscr{T}$, the RGF method can be used to calculate other quantities such as the local density of states (e.g. [41]) and the local current density (e.g. [42,43]).

\subsection{Local density of states}

The local density of states (LDOS) can be easily evaluated if one knows the exact retarded Green's function at a given site:

$\rho(n, j ; E)=-\frac{1}{\pi} \operatorname{Im}\left[G_{n, n}^{r}(j, j ; E)\right]$.

The exact local Green's function is evaluated using Eq. (41). Conductance calculations require just a single sweep through the lattice, since the transmission formula (15) only needs $G^{r}$ evaluated at the slices corresponding to the contacts. In contrast, the LDOS demands the calculation of $G^{r}$ at all lattice slices of interest, which further increases the computational cost linearly with $N$, resulting in $O\left(N^{2} M^{3}\right)$. Let us 
provide an example of a situation where the LDOS plays a central role and needs to be evaluated.

Numerous studies have addressed the possibility of local magnetic moment formation in graphene either due to zigzag terminations at the edges in graphene nanoribbons or due to vacancies in graphene sheets (for a review, see Ref. [44]). The effect can be understood through the Stoner mechanism of magnetism, which requires an enhanced LDOS when strong electron-electron interaction as present, as originally proposed in Ref. [45] using the Density Functional Theory (DFT). The tight-binding Hamiltonian, Eq. (4), can be modified to reproduce the DFT results by adding a Hubbard mean-field term [45], namely,

$$
\begin{aligned}
H=-\sum_{i<j, \sigma} & \left(t_{i j} c_{i, \sigma}^{\dagger} c_{j, \sigma}+\text { H.c. }\right) \\
& +\sum_{i, \sigma}\left(V_{i}+U\left\langle n_{i,-\sigma}\right\rangle\right) c_{i, \sigma}^{\dagger} c_{i, \sigma},
\end{aligned}
$$

where the operators $c_{i, \sigma}^{\dagger}$ and $c_{i, \sigma}$ create and annihilate an electron of spin projection $\sigma$ at at the site $i$. As standard, $\left\langle n_{i, \sigma}\right\rangle$ is the occupation number and $U$ is the on-site electronelectron interaction strength. In graphene, $U$ is usually fitted to reproduce the DFT band structure calculations for translation invariant systems (for which $V_{i}$ is constant). As we discuss in Sec. 8, by a suitable choice of the parameters $V_{i}$ and $t_{i j}$, the Hamiltonian 107 becomes an excellent framework to model disorder as well.

In general, the occupation numbers $\left\langle n_{i, \sigma}\right\rangle$ that appear in Eq. 107) can be obtained from nonequilibrium Green's functions [31]. In the linear response regime, a simplification allows one to express $\left\langle n_{i, \sigma}\right\rangle$ in terms of an equilibrium Green's function, namely,

$\left\langle n_{i, \sigma}\right\rangle=-\frac{1}{\pi} \int_{-\infty}^{\infty} d E \operatorname{Im}\left[G_{n, n ; \sigma}^{r}(j, j ; E)\right] f(E-\mu)$.

where $i \equiv(j, n)$. Note that Eqs. (107) and 108) have to be solved self-consistently.

The large number of poles of $G^{r}(E)$ makes impractical the integration of the r.h.s. of Eq. 108 on the real axis, a difficulty shared with transport studies on molecular electronics, see e.g. Ref. [46]. An optimized strategy to implement an efficient integration along a complex plane contour is presented in Refs. [34,47.

Another frequent application of LDOS occurs in the evaluation of the local charge density. The latter can be calculated from the LDOS through the expression

$n_{c}\left(n, j ; E_{F}\right)=\frac{1}{A} \int_{\mu(n, j)}^{E_{F}} d E \rho(n, j ; E)$,

where $A$ is the sheet area and $\mu(n, j)$ denotes the local chemical potential. Note that in $n$-type regions, $E_{F}>\mu$ and therefore the integral is over positive energies ("electrons"), while in $p$-type regions, $E_{F}<\mu$ and the integral is over negative energies ("holes"). The local chemical potential is evaluated with respect to $E_{F}=0$,

$\mu(n, j)=V(n, j)$,

where the background potential $V(n, j)$ includes the gate voltage.

\subsection{Local current density}

The local current density is involves more complex calculation than the LDOS. Several methods were developed in the literature (see e.g. Ref. [42]). The bond current between two neighboring sites of lattice coordinates $(n, j)$ and $\left(n^{\prime}, j^{\prime}\right)$ is obtained using the equations-of-motion method for nonequilibrium Green's functions (see e.g. [31]) and reads [24]

$$
\begin{aligned}
I_{(n, j) \rightarrow\left(n^{\prime}, j^{\prime}\right)}=-\frac{2 e}{h} \int d E[ & U_{n, n^{\prime}}\left(j, j^{\prime}\right) G_{n^{\prime}, n}^{<}\left(j^{\prime}, j\right) \\
& \left.\quad-U_{n^{\prime}, n}\left(j^{\prime}, j\right) G_{n, n^{\prime}}^{<}\left(j, j^{\prime}\right)\right],
\end{aligned}
$$

where $G^{<}$is the lesser Green's function of the system. 2 For the tight-binding model with nearest-neighbor hopping, there are two situations to consider. First, $n=n^{\prime}$, in which case $j^{\prime}=j \pm 1$ and the current is intra-slice. Second, when $n^{\prime}=n \pm 1$, the current is inter-slice and $j^{\prime}=j$ or $j^{\prime}=j \pm 1$, at most. Notice that in the absence of magnetic fields, timereversal symmetry requires $U_{n, n^{\prime}}\left(j, j^{\prime}\right)=U_{n^{\prime}, n}\left(j^{\prime}, j\right)$. In addition, in equilibrium conditions, the lesser Green's function is a symmetric matrix, leading to a zero bond current, as expected. Thus, local currents can only appear through the application of a magnetic field (which breaks time-reversal symmetry) or when a finite bias voltage between contacts exists.

Equation (111) requires the calculation of the exact lesser Green's function for sites in the bulk of the system. This can be done recursively, starting from the equilibrium Green's function of the leads (see Sec.6.3). An alternative approach, suitable for transport in the linear regime, is to use the elimination technique developed in Ref. [43], where only retarded and advanced Green's functions are required and the energy integration is avoided. The computational cost is this approach also scales as $O\left(N^{2} M^{3}\right)$.

For plotting current fields, it is useful to define the local, on-site, outgoing vector current as

$\mathbf{I}_{n, j}=\sum_{n^{\prime}, j^{\prime}} \mathbf{a}_{(n, j) \rightarrow\left(n^{\prime}, j^{\prime}\right)} I_{(n, j) \rightarrow\left(n^{\prime}, j^{\prime}\right)}$,

2 There are several good textbooks, such as Refs. [31.48 49], that discuss nonequilibrium Green's functions. In particular, Ref. [31] concisely covers all required background material. We refer the reader to these books for the derivation of expressions involving $G^{<}$and related functions and further insight into the subject. 
where the sum is over sites $\left(n^{\prime}, j^{\prime}\right)$ that are nearest neighbors to site $(n, j)$ and $\mathbf{a}_{k, k^{\prime}}$ is the lattice vector between sites $k$ and $k^{\prime}$. Notice that this local vector current does not necessarily fall along any of the bonds coming out of the site $(n, j)$.

\subsection{Recursion for non-equilibrium Green's functions}

In order to evaluate the local current distribution 1111 in the most general case, one needs to determine the exact lesser Green's function, $G^{<}$. The procedure is the following.

1. We start with the retarded Green's functions of the leads (which are assumed to be in equilibrium) and use the fluctuation-dissipation relations

$$
g_{L}^{<}=-i f_{L}\left(g_{L}^{r}-g_{L}^{a}\right),
$$

and

$$
g_{R}^{<}=-i f_{R}\left(g_{R}^{r}-g_{R}^{a}\right),
$$

where the advanced Green's functions obey $g_{L}^{a}=\left(g_{L}^{r}\right)^{\dagger}$ and $g_{R}^{a}=\left(g_{R}^{r}\right)^{\dagger}$. Here, $f_{L}$ and $f_{R}$ denote the Fermi distributions in the left and right leads, respectively.

2. For the left-to-right sweep, we first determine the retarded Green's function at the $n$th slice and then obtain the lesser Green's function using the expression

$G_{n, n}^{L,<}=G_{n, n}^{L, r} \Sigma_{n, n}^{L,<} G_{n, n}^{L, a}$,

where the self energy due to the coupling of the $n$th slice to all other slices to the left is given by

$\Sigma_{n, n}^{L,<}=U_{n, n-1} G_{n-1, n-1}^{L,<} U_{n-1, n}$.

Notice that $G_{n, n}^{L, a}=\left(G_{n, n}^{L, r}\right)^{\dagger}$ and $U_{n, n-1}=\left(U_{n-1, n}\right)^{\dagger}$. Thus, we obtain the recurrence relation

$G_{n, n}^{L,<}=\left(G_{n, n}^{L, r} U_{n, n-1}\right) G_{n-1, n-1}^{L,<}\left(G_{n, n}^{L, r} U_{n, n-1}\right)^{\dagger}$.

3. For the right-to-left sweep, we apply instead the analogous expression

$G_{n, n}^{R,<}=G_{n, n}^{R, r} \Sigma_{n, n}^{R,<} G_{n, n}^{R, a}$,

where

$\Sigma_{n, n}^{R,<}=U_{n, n+1} G_{n+1, n+1}^{R,<} U_{n+1, n}$.

Thus, we obtain the other recurrence relation

$G_{n, n}^{R,<}=\left(G_{n, n}^{R, r} U_{n, n+1}\right) G_{n+1, n+1}^{R,<}\left(G_{n, n}^{R, r} U_{n, n+1}\right)^{\dagger}$.

4. In order to join the two sweeps to obtain the exact lesser Green's function at a given slice, we simply combine left and right self energies and evaluate

$G_{n, n}^{<}=G_{n, n}^{r}\left(\Sigma_{n, n}^{L,<}+\Sigma_{n, n}^{R,<}\right) G_{n, n}^{a}$

using Eqs. 1116 and 119 for the self energies.
5. Once the left-to-right and right-to-left Green's functions are know, the exact inter-slice lesser Green's functions are obtained using the Dyson-Langreth equations [31]

$$
G_{n-1, n}^{<}=G_{n-1, n-1}^{L, r} U_{n-1, n} G_{n, n}^{<}+G_{n-1, n-1}^{L,<} U_{n-1, n} G_{n, n}^{a},
$$

$G_{n, n+1}^{<}=G_{n, n}^{r} U_{n, n+1} G_{n+1, n+1}^{R,<}+G_{n, n}^{<} U_{n, n+1} G_{n+1, n+1}^{R, a}$,

$G_{n, n-1}^{<}=G_{n, n}^{r} U_{n, n-1} G_{n-1, n-1}^{L,<}+G_{n, n}^{<} U_{n, n-1} G_{n-1, n-1}^{L, a}$,

and

$G_{n+1, n}^{<}=G_{n+1, n+1}^{R, r} U_{n+1, n} G_{n, n}^{<}+G_{n+1, n+1}^{R,<} U_{n+1, n} G_{n, n}^{a}$.

\subsection{Current conservation}

Notice that the current through consecutive slices is conserved. Let us prove that using Eq. 111 and writing

$I_{(n-1) \rightarrow n}=-\frac{e}{h} \int d E \operatorname{Tr}\left(G_{n, n-1}^{<} U_{n-1, n}-U_{n, n-1} G_{n-1, n}^{<}\right)$

and

$I_{n \rightarrow(n+1)}=-\frac{e}{h} \int d E \operatorname{Tr}\left(G_{n+1, n}^{<} U_{n, n+1}-U_{n+1, n} G_{n, n+1}^{<}\right)$,

where the traces indicate a sum over all sites in the slices $n$ and $n+1$, respectively. Since

$G_{n, n-1}^{<} U_{n-1, n}=G_{n, n}^{r} \Sigma_{n, n}^{L,<}+G_{n, n}^{<} \Sigma_{n, n}^{L, a}$,

$U_{n, n-1} G_{n-1, n}^{<}=\Sigma_{n, n}^{L, r} G_{n, n}^{<}+\Sigma_{n, n}^{L,<} G_{n, n}^{a}$,

$G_{n, n+1}^{<} U_{n+1, n}=G_{n, n}^{r} \Sigma_{n, n}^{R,<}+G_{n, n}^{<} \Sigma_{n, n}^{R, a}$,

and

$U_{n, n+1} G_{n+1, n}^{<}=\sum_{n, n}^{R, r} G_{n, n}^{<}+\Sigma_{n, n}^{R,<} G_{n, n}^{a}$,

it is straightforward to show that $I_{(n-1) \rightarrow n}=I_{n \rightarrow(n+1)}$, which guarantees current conservation. It is also possible to show that the total current leaving any site is zero. 


\section{Dephasing}

The model Hamiltonian (4) describes electrons within the single-particle approximation, disregarding the effects of electron-phonon and electron-electron interactions. Let us call as "environment" all the degrees of freedom that couple to electrons in the real physical device. Even at very low temperatures, due to the interaction with the environment, the quantum interference between different electronic paths typically fades away at lengths larger than the scale $\ell_{\varphi}$. The latter is called coherence length or dephasing length. Currently, $\ell_{\varphi}$ in graphene experiments can be as high as few microns at low temperatures, decreasing with increasing temperature.

This Section shows how to incorporate dephasing into the RGF method. We follow the phenomenological approach pioneered by Büttiker [50] and D'Amato and Pastawski [51] and introduce dephasing in the calculations by adding a set of voltage probes to the system. These voltage probes act on selected system sites and have their individual chemical potentials adjusted as not drain or inject any net current. The voltage probes give rise to dephasing because the drained electrons are not phase coherent with the ones injected back. The dephasing length $\ell_{\varphi}$ is related to the number of voltage probes and the strength of their coupling to the system. We focus on the linear transport regime and assume that all scattering within the voltage probes, albeit incoherent, is elastic. Therefore we neglect any "vertical flow", as defined by Datta [21].

The basic linear response equations are

$$
\begin{aligned}
& I_{L}=\mathscr{Q}_{L L} \mu_{L}-\mathscr{Q}_{L R} \mu_{R}-\sum_{i=1}^{N_{\varphi}} \mathscr{Q}_{L i} \mu_{i}, \\
& I_{R}=\mathscr{Q}_{R R} \mu_{R}-\mathscr{Q}_{R L} \mu_{L}-\sum_{i=1}^{N_{\varphi}} \mathscr{Q}_{R i} \mu_{i}, \\
& I_{i}=\mathscr{Q}_{i i}^{\varphi} \mu_{i}-\sum_{i^{\prime}=1\left(i^{\prime} \neq i\right)}^{N_{\varphi}} \mathscr{Q}_{i i^{\prime}}^{\varphi} \mu_{i^{\prime}}-\mathscr{Q}_{i R} \mu_{R}-\mathscr{Q}_{i L} \mu_{L},
\end{aligned}
$$

where $\mu_{R(L)}$ denotes the chemical potential in the right (left) lead and $\mu_{i}$ is the chemical potential of the $i$ th voltage probe, $i=1, \ldots N_{\varphi}$, where $N_{\varphi}$ is the total number of voltage probes. The linear transport coefficients $\mathscr{Q}$ need to be determined (see below). The left, right, and probe currents $I_{L}, I_{R}$, and $I_{i}$, respectively, are defined as positive when they flow into the system. Since there is no net probe current, we set $I_{i}=0$ for all probes. Therefore,

$$
\mathscr{Q}_{i i}^{\varphi} \mu_{i}=\sum_{i^{\prime}=1\left(i^{\prime} \neq i\right)}^{N_{\varphi}} \mathscr{Q}_{i i^{\prime}}^{\varphi} \mu_{i^{\prime}}+\mathscr{Q}_{i L} \mu_{L}+\mathscr{Q}_{i R} \mu_{R}
$$

In addition, notice that when all the chemical potentials are identical, all currents should vanish. Thus,

$$
\begin{array}{r}
\sum_{i=1}^{N_{\varphi}} \mathscr{Q}_{L i}+\mathscr{Q}_{L R}-\mathscr{Q}_{L L}=0, \\
\sum_{i=1}^{N_{\varphi}} \mathscr{Q}_{R i}+\mathscr{Q}_{R L}-\mathscr{Q}_{R R}=0, \\
\mathscr{Q}_{i i}^{\varphi}-\sum_{i^{\prime}=1\left(i^{\prime} \neq i\right)}^{N_{\varphi}} \mathscr{Q}_{i i^{\prime}}^{\varphi}-\mathscr{Q}_{i L}-\mathscr{Q}_{i R}=0 .
\end{array}
$$

Using Eqs. 135 and 138, we can then write

$$
\sum_{i^{\prime}=1}^{N_{\varphi}} \mathscr{W}_{i i^{\prime}}^{\varphi}\left(\mu_{i^{\prime}}-\mu_{R}\right)=\mathscr{Q}_{i L}\left(\mu_{L}-\mu_{R}\right),
$$

where $\mathscr{W}_{i i}=\mathscr{Q}_{i i}^{\varphi}$ and $\mathscr{W}_{i i^{\prime}}=-\mathscr{Q}_{i i^{\prime}}^{\varphi}$ if $i \neq i^{\prime}$. Whenever the matrix $\mathscr{W}$ is invertible, we obtain

$\mu_{i}=\mu_{R}+\sum_{i^{\prime}=1}^{N_{\varphi}}\left(\mathscr{W}^{-1}\right)_{i i^{\prime}} \mathscr{Q}_{i^{\prime} L}\left(\mu_{L}-\mu_{R}\right)$.

Substituting Eqs. (137) and (140) into Eq. 133), we have

$I_{R}=\overline{\mathscr{Q}}_{R L}\left(\mu_{R}-\mu_{L}\right)$,

where

$\overline{\mathscr{Q}}_{R L}=\mathscr{Q}_{R L}+\sum_{i, i^{\prime}=1}^{N_{\varphi}} \mathscr{Q}_{R i}\left(\mathscr{W}^{-1}\right)_{i i^{\prime}} \mathscr{Q}_{i^{\prime} L}$.

Notice that we have expressed the right-lead current (which is equal to minus the left-lead current) in terms of the difference between the chemical potential in the leads.

The linear transport coefficients entering in Eq. (142) can be obtained from the conductance matrix of the system:

$$
\begin{aligned}
|e| \mathscr{Q}_{R L} & =\mathscr{G}_{R L}, \\
|e| \mathscr{Q}_{R i} & =\mathscr{G}_{R i}, \\
|e| \mathscr{Q}_{i L} & =\mathscr{G}_{i L}, \\
|e| \mathscr{Q}_{i i^{\prime}}^{\varphi} & =\mathscr{G}_{i i^{\prime}}, \quad i \neq i^{\prime} \\
|e| \mathscr{Q}_{i i}^{\varphi} & =\sum_{i^{\prime}=1\left(i^{\prime} \neq i\right)}^{N_{\varphi}} \mathscr{G}_{i i^{\prime}}+\mathscr{G}_{i L}+\mathscr{G}_{i R} .
\end{aligned}
$$

(This makes $\sum_{i=1}^{N_{\varphi}} \mathscr{W}_{i i^{\prime}} \neq 0$, thus $\mathscr{W}$ is in principle invertible.) The coefficients $\mathscr{W}$ are positive. This is consistent with the assumption that currents run from higher to lower chemical potential. The conductances are calculated using the Caroli formulas

$$
\begin{aligned}
\mathscr{G}_{R L} & =\frac{2 e^{2}}{h} \operatorname{Tr}\left[\Gamma_{R} G_{N+1,0}^{r} \Gamma_{L} G_{0, N+1}^{a}\right], \\
\mathscr{G}_{R i} & =\frac{2 e^{2}}{h} \operatorname{Tr}\left[\Gamma_{R} G_{N+1, n_{i}}^{r} \Gamma_{i} G_{n_{i}, N+1}^{a}\right], \\
\mathscr{G}_{i L} & =\frac{2 e^{2}}{h} \operatorname{Tr}\left[\Gamma_{i} G_{n_{i}, 0}^{r} \Gamma_{L} G_{0, n_{i}}^{a}\right], \\
\mathscr{G}_{i i^{\prime}} & =\frac{2 e^{2}}{h} \operatorname{Tr}\left[\Gamma_{i} G_{n_{i}, n_{i^{\prime}}}^{r} \Gamma_{i^{\prime}} G_{n_{i^{\prime}}, n_{i}}^{a}\right] .
\end{aligned}
$$


Here, we use the pair $\left(n_{i}, j_{i}\right)$ to denote the site coordinates of the $i$ th voltage probe. The level width probe matrix $\Gamma_{i}$ can be obtained much in the same way as $\Gamma_{R}$ and $\Gamma_{L}$, namely, from the probe surface Green's function (see Sec. 2). Notice that in the absence of magnetic fields, all cross-conductance matrices are symmetric. In addition, since the coupling matrices $\Gamma_{R}, \Gamma_{L}$, and $\Gamma_{i}$ are all Hermitian and positive, one can easily show that these conductances are all real and positive.

In practice, we will assume that the set of lattice points attached to voltage probes is sparse, so that $N_{\varphi} \ll N M$ and the computation cost of evaluating the cross conductances is not too high. The number of voltage probes $N_{\varphi}$ and the magnitude of the $\Gamma_{i}$ 's (see Sec.7.2) determine $\ell_{\varphi}$.

\subsection{Self consistency}

The Green's function entering in Eqs. (148) to Eq. (151) are the exact ones. Therefore, they have to take into account the coupling to the voltage probes and, consequently, should depend on the chemical potentials $\left\{\mu_{i}\right\}$. These, however, depend on the left and right chemical potentials and on the cross-conductance matrices $\mathscr{Q}$, which are given by Eqs. (144) to (146). Thus, one can see that this calculation needs to be implemented self-consistently. There is one trivial case though, namely, when the bias across the system is zero and $\mu_{R}=\mu_{L}$ (equilibrium condition). In this case, Eq. (140) shows that all $\mu_{i}=\mu_{R}$ and the self-consistency can be trivially satisfied. Nevertheless, one still needs to include the self-energy of the voltage probes in the local Green's function of each slice during the recursive calculation: $h_{n} \rightarrow$ $h_{n}+\Sigma_{i}$, when $i \in n$.

\subsection{Voltage probe self energy}

The voltage probe partial width $\Gamma_{i}$ is related to the voltage probe self energy $\Sigma_{i}$ in the standard way: $\Gamma_{i}=i\left(\Sigma_{i}^{r}-\Sigma_{i}^{a}\right)$. When each voltage probe is attached a single site, $\Gamma_{i}$ and $\Sigma_{i}^{r}$ are just complex numbers and the insertion of the probe self energy into the calculation is substantially simplified. For a one-dimensional semi-infinite chain with hopping matrix element $t$ coupled to a system site through a hopping amplitude $t_{\varphi}$, the retarded Green's function reads (see Sec.4.1)

$g_{i}^{r}(E)=\frac{E-\mu_{i}}{2 t^{2}}\left[1-\sqrt{1-\frac{4 t^{2}}{\left(E-\mu_{i}\right)^{2}}}\right]$

Thus, the retarded surface self energy of the $i$ th probe is equal to

$\Sigma_{i}^{r}(E)=t_{\varphi}^{2} g_{i}^{r}(E)=\left(\frac{t_{\varphi}}{t}\right)^{2} \frac{E-\mu_{i}}{2}\left[1-\sqrt{1-\frac{4 t^{2}}{\left(E-\mu_{i}\right)^{2}}}\right]$, leading to

$\Gamma_{i}(E)= \begin{cases}0, & \left|E-\mu_{i}\right|>2 t, \\ \left(\frac{t_{\varphi}}{t}\right)^{2} \sqrt{4 t^{2}-\left(E-\mu_{i}\right)^{2}}, & \left|E-\mu_{i}\right|<2 t .\end{cases}$

The retarded Green's function of a slice which is isolated from other slices should include the self energy of any attached voltage probe:

$\left[g_{n}^{-1}\right]\left(j, j^{\prime}\right)=\left\{\begin{array}{ll}E-h_{n}(j, j)-\Sigma_{i}^{r}(E), & j=j^{\prime}, \\ E-h_{n}\left(j, j^{\prime}\right)+i 0^{+}, & j \neq j^{\prime}\end{array}\right.$,

when $n_{i}=n$ and $j=j_{i}$.

\subsection{Recursion for dephasing Green's functions}

In order to evaluate the cross-conductance matrices that enter in Eq. 143, it is necessary to evaluate the exact Green's function between two slices carrying voltage probes, say, $n_{1}$ and $n_{2}$; for instance, see Eq. 151). This is a computationally intensive task and can only be carried out in a relatively efficient way if all left-to-right or right-to-left local Green's functions are stored during sweeps and the exact local Green's functions at either $n_{1}$ or $n_{2}$ have already been calculated and stored.

Suppose that we want to evaluate the retarded Green's function $G_{n_{1}, n_{2}}$, with $n_{1}<n_{2}$. Here are the steps:

1. Starting with $G_{n_{1}, n_{1}}^{L}$ and $G_{n_{1}+1, n_{1}+1}^{L}$ obtained during the left-to-right sweep, evaluate

$$
G_{n_{1}, n_{1}+1}^{L}=G_{n_{1}, n_{1}}^{L} U_{n_{1}, n_{1}+1} G_{n_{1}+1, n_{1}+1}^{L} .
$$

2. Next, use the resulting Green's function and the stored $G_{n_{1}+2, n_{1}+2}^{L}$ to evaluate

$$
G_{n_{1}, n_{1}+2}^{L}=G_{n_{1}, n_{1}+1}^{L} U_{n_{1}+1, n_{1}+2} G_{n_{1}+2, n_{1}+2}^{L} .
$$

3. Repeat this procedure until $G_{n_{1}, n_{2}-1}^{L}$ is obtained.

4. From the previously calculated $G_{n_{2}, n_{2}}$, evaluate

$$
G_{n_{1}, n_{2}}=G_{n_{1}, n_{2}-1}^{L} U_{n_{2}-1, n_{2}} G_{n_{2}, n_{2}} .
$$

Likewise, for $n_{1}>n_{2}$, we follow these steps:

1. Starting with $G_{n_{1}, n_{1}}^{R}$ and $G_{n_{1}-1, n_{1}-1}^{R}$ obtained during the right-to-left sweep, evaluate

$$
G_{n_{1}, n_{1}-1}^{R}=G_{n_{1}, n_{1}}^{R} U_{n_{1}, n_{1}-1} G_{n_{1}-1, n_{1}-1}^{R} .
$$

2. Next, use the resulting Green's function and the stored $G_{n_{1}-2, n_{1}-22}^{L}$ to evaluate

$$
G_{n_{1}, n_{1}-2}^{R}=G_{n_{1}, n_{1}-1}^{R} U_{n_{1}-1, n_{1}-2} G_{n_{1}-2, n_{1}-2}^{R} .
$$

3. Repeat this procedure until $G_{n_{1}, n_{2}+1}^{R}$ is obtained.

4. From the previously calculated $G_{n_{2}, n_{2}}$, evaluate

$$
G_{n_{1}, n_{2}}=G_{n_{1}, n_{2}+1}^{R} U_{n_{2}+1, n_{2}} G_{n_{2}, n_{2}} \text {. }
$$

Since no inversions are required in these steps, the calculation scales as $O\left(\left|n_{1}-n_{2}\right| M\right)$. 


\section{Disorder}

Disorder is ubiquitous in graphene samples, even in those synthesized with state-of-the-art technologies. Depending on the synthesis method, charge density inhomogeneities [52] or substrate irregularities [53], intrinsic and extrinsic ripples [54,55], strain fields [56], surface molecular adsorption [57], vacancies [58], and irregular edges [59], are unavoidable. The effects of these different kinds of disorder on transport in graphene have been addressed by several reviews [1, [2,60,61] without exhausting the subject.

The RGF method is flexible enough to address any of the above-mentioned types of disorder by a suitable choice of the hopping $t_{i j}$ and the local potential $V_{i}$ in the Hamiltonian (4). Since it is based on an atomistic basis, the RGF method is ideal for studying numerically short-range disorder effects, whose typical range is of the order of the lattice spacing. Although not optimized for that purpose, the method can also be used to address long-range disorder 40 , 30, 62, 63.

In this Section we present some common disorder models for graphene and discuss their implementation within the RGF method.

\subsection{Diagonal (scalar) disorder}

Charge density and substrate inhomogeneities can be modeled by adding a local disordered potential $U\left(\mathbf{r}_{i}\right)$ to the lattice sites in the sample region. One of the simplest models for $U\left(\mathbf{r}_{i}\right)$ is constructed as follows: We take $\mathscr{N}_{\text {imp }}$ random lattice sites $\left\{\mathbf{R}_{k}\right\}$ uniformly distributed as centers of Gaussian scatterers with a random amplitude $U_{k}$ taken from a uniform distribution over the interval $[-\delta V, \delta V]$. This results in

$U\left(\mathbf{r}_{n, j}\right)=\sum_{k=1}^{\mathscr{N}_{\text {imp }}} U_{k} e^{-\left|\mathbf{r}_{n, j}-\mathbf{R}_{k}\right|^{2} / 2 \xi^{2}}$

where $\xi$ is the range of the potential [3, 40]. The concentration of scatterers is $n_{\text {imp }}=\mathscr{N}_{\text {imp }} / \mathscr{A}$, where $\mathscr{A}$ denotes the total area of the sample.

In the limit of a low concentration of scatterers, $n_{\mathrm{imp}}^{-1 / 2} \gg$ $\xi$, the magnitude of the disorder fluctuations is characterized by the dimensionless parameter $K_{0}$, which is defined from the impurity potential correlation function,

$\left\langle U\left(\mathbf{r}_{n, j}\right) U\left(\mathbf{r}_{n^{\prime}, j^{\prime}}\right)\right\rangle=\frac{K_{0}(\hbar v)^{2}}{2 \pi \xi^{2}} e^{-\left|\mathbf{r}_{n, j}-\mathbf{r}_{n^{\prime}, j^{\prime}}\right|^{2} / 4 \xi^{2}}$,

where $v=\sqrt{3} a_{0} t / 2 \hbar$ is the Fermi velocity and $\langle\cdots\rangle$ stands for the average over disorder realizations. It is easy to see that $\left\langle U\left(\mathbf{r}_{n, j}\right)\right\rangle=0$. We note that $K_{0}$ contains information not only about the relative magnitude of the potential fluctuations, $\delta V / t$, but also about the scatterers' range and concentration: A simple calculation yields [40]

$K_{0} \approx \frac{16 \pi^{2}}{9} n_{\text {imp }}\left(\frac{\delta V}{t}\right)^{2}\left(\frac{\xi^{4}}{a_{0}^{2}}\right)$.

If we now recall that there are two inequivalent atoms per hexagon and $A_{\text {hex }}=\sqrt{3} a_{0}^{2} / 2$, we find that

$K_{0} \approx \frac{64 \pi^{2}}{9 \sqrt{3}} \frac{\mathscr{N}_{\mathrm{imp}}}{\mathscr{N}}\left(\frac{\delta V}{t}\right)^{2}\left(\frac{\xi}{a_{0}}\right)^{4}$,

where the numerical prefactor is approximately 40.5 and $\mathscr{N}$ is the total number of lattice sites.

In the continuum limit and using Eq. (163) together with the Born approximation (BA), one finds that the transport mean free path away from the Dirac point is given by

$\ell_{\mathrm{tr}}^{\mathrm{BA}}=\frac{2}{\pi} \frac{\lambda_{F}}{K_{0}}$

where $\lambda_{F}$ is the Fermi wavelength in the graphene sheet [3] $\left(\lambda_{F} \ll \ell_{\mathrm{tr}}^{\mathrm{BA}}\right.$ for the BA to hold).

\subsection{Off-diagonal disorder - strain}

Let us consider the situations where the graphene sheet is subjected to strain. We now address the case where the strain field modifies the carbon-carbon bond lengths, postponing to the next subsection the discussion of bond distortion due to curvatures.

Modifications in the bond lengths lead to a hopping renormalization. In the Slater-Koster scheme, the carbon-carbon hopping term can, in principle, be obtained from the dependence of the $V_{p p \pi}$ on the inter-orbital distance. In practice, one relies on semi-empirical parameterizations, such as [64]

$V_{p p \pi}(l)=-t e^{-3.37(l / a-1)}$

where $l$ is the bond length and $a$ is the inter-atomic distance in the honeycomb lattice. The decay rate is adjusted to fit the experimental result $d V_{p p \pi} / d l=-6.4 \mathrm{eV} / \AA$. With the help of Eq. 167, local bond length deformations $\delta l_{i, j}=l_{i, j}-a$ are translated into changes in the hopping integrals. The latter are easily accounted for by the RGF method.

The macroscopic theory of elasticity can help to translate the strain field acting on a graphene sheet into modifications of the hopping integrals. That is because the tensions along the graphene membrane change very slowly on the microscopic scale. Hence, the changes in the bond lengths $\delta l_{i, j}$ can be approximated by a smooth function $\delta l(x, y)$, where $(x, y)$ is the position of the $i$ th site in the honeycomb lattice. In turn, the $\delta l(x, y)$ can be related to the strain fields by the elastic theory [65]. Reference [64], for instance, writes the strain tensor $\varepsilon$ for the case of uniaxial strain and shows how to relate $\varepsilon$ to the bond length deformations. 
8.3 Off-diagonal disorder - ripples

Let us assume that there is a ripple structure in the graphene sheet. The ripples can be described by a scalar field $h(x, y)$ which represents the out-of-plane displacement of the carbon atoms at a given location $(x, y)$. A non-homogeneous $h(x, y)$ ripple-field modifies the atomic orbital overlaps and, hence, the hopping terms in the tight-binding model.

Neglecting bond length stretching due to strain, the ripples affect the nearest neighbor and next-to-nearest neighbor hopping matrix elements: $t_{i j}=t_{i j}^{(0)}+\delta t_{i, j}$, where $t_{i j}^{(0)}$ is the hopping between sites $i$ and $j$ in the absence of ripples and [66]

$\delta t_{i j} \approx-\frac{1}{2} E_{i j}\left[\left(\mathbf{u}_{i j} \cdot \nabla\right) \nabla h\right]^{2}$.

Here, $\mathbf{u}_{i j}$ is the unit vector connecting sites $i$ and $j$ and $E_{i j}=$ $t_{i j}^{(0)} / 3+V_{p p \sigma ; i j} / 2$, where $V_{p p \sigma ; i j}$ describes the overlap of the $\sigma$-orbitals (the effect of the $\sigma$ orbitals is only negligible in the absence of bending). The scales are the following:

$t_{i j}^{(0)}= \begin{cases}-2.7 \mathrm{eV}, & \text { for n.n. } \\ -0.1 \mathrm{eV}, & \text { for n.n.n., }\end{cases}$

and

$V_{p p \sigma ; i j}= \begin{cases}5.8 \mathrm{eV}, & \text { for n.n. } \\ 1.4 \mathrm{eV}, & \text { for n.n.n.. }\end{cases}$

The free energy associated to the ripple field $h(\mathbf{r})$ for graphene on a substrate is given by the Gaussian (elastic) form 66

$F=\frac{1}{2} \int d^{2} r\left[\kappa\left(\nabla^{2} h\right)^{2}+\gamma(\nabla h)^{2}+v(h-s)^{2}\right]$,

where $\kappa$ is the bending rigidity, $\gamma$ is the interfacial stiffness, and $v$ is a coupling constant for the pinning of the graphene sheet by the background roughness $s(x, y)$. Typically, $\kappa \approx 1$ $\mathrm{eV}$. The other two parameters, $\gamma$, and $v$, will depend on the substrate.

In order to generate the appropriate random $h(\mathbf{r})$, we will assume that the temperature is low and thermal fluctuations can be neglected (this hypothesis could in principle be relaxed). In this case, the fluctuations in $h(\mathbf{r})$ follow those of the substrate: Minimizing the free energy in Eq. (171), we obtain

$\left(\kappa \nabla^{4}-\gamma \nabla^{2}+v\right) h(x, y)=v s(x, y)$.

Using the Fourier decompositions $h(\mathbf{r})=\sum_{\mathbf{q}} \tilde{h}(\mathbf{q}) e^{i \mathbf{q} \cdot \mathbf{r}}$ and $s(\mathbf{r})=\sum_{\mathbf{q}} \tilde{s}(\mathbf{q}) e^{i \mathbf{q} \cdot \mathbf{r}}$ we obtain

$\left(\kappa q^{4}+\gamma q^{2}+v\right) \tilde{h}(\mathbf{q})=v \tilde{s}(\mathbf{q})$.

As a result,

$\left\langle\tilde{h}\left(\mathbf{q}_{1}\right) \tilde{h}\left(\mathbf{q}_{2}\right)\right\rangle=\frac{v^{2}\left\langle\tilde{s}\left(\mathbf{q}_{1}\right) \tilde{s}\left(\mathbf{q}_{2}\right)\right\rangle}{\left(\kappa q_{1}^{4}+\gamma q_{1}^{2}+v\right)\left(\kappa q_{2}^{4}+\gamma q_{2}^{2}+v\right)}$.
If the background has white-noise fluctuations, $\left\langle\tilde{s}\left(\mathbf{q}_{1}\right) \tilde{s}\left(\mathbf{q}_{2}\right)=\right.$ $s_{0}^{2} \boldsymbol{\delta}^{(2)}\left(\mathbf{q}_{1}+\mathbf{q}_{2}\right)$, we get

$\left\langle\tilde{h}\left(\mathbf{q}_{1}\right) \tilde{h}\left(\mathbf{q}_{2}\right)\right\rangle=\frac{v^{2} s_{0}^{2} \delta^{(2)}\left(\mathbf{q}_{1}+\mathbf{q}_{2}\right)}{\left(\kappa q_{1}^{4}+\gamma q_{1}^{2}+v\right)^{2}}$.

Since

$\langle h(\mathbf{r}) h(0)\rangle=\sum_{\mathbf{q}_{1}, \mathbf{q}_{2}}\left\langle\tilde{h}\left(\mathbf{q}_{1}\right) \tilde{h}\left(\mathbf{q}_{2}\right)\right\rangle e^{i \mathbf{q}_{1} \cdot \mathbf{r}}$,

we finally arrive at

$\langle h(\mathbf{r}) h(0)\rangle=\sum_{\mathbf{q}} \frac{v^{2} s_{0}^{2}}{\left(\kappa q^{4}+\gamma q^{2}+v\right)^{2}} e^{i \mathbf{q} \cdot \mathbf{r}}$.

We now need to find a way to generate membrane profiles $h(x, y)$ that satisfy this correlation function. The solution is simple: Let us introduce

$h_{c}(\mathbf{r})=\sum_{\mathbf{q}} \frac{v s_{0}}{\kappa q^{4}+\gamma q^{2}+v} e^{i\left(\mathbf{q} \cdot \mathbf{r}+\phi_{\mathbf{q}}\right)}$,

where the phases $\left\{\phi_{\mathbf{q}}\right\}$ are uniformly distributed in the interval $[0: 2 \pi)$ and uncorrelated, except that $\phi_{\mathbf{q}}=-\phi_{-\mathbf{q}}$ in order to define a real $h_{c}$. It then follows that

$$
\begin{aligned}
\left\langle h_{c}(\mathbf{r}) h_{c}(0)\right\rangle= & \sum_{\mathbf{q}_{1}, \mathbf{q}_{2}} \frac{v^{2} s_{0}^{2}}{\left(\kappa q_{1}^{4}+\gamma q_{1}^{2}+v\right)\left(\kappa q_{2}^{4}+\gamma q_{2}^{2}+v\right)} \\
& \times e^{i \mathbf{q}_{1} \cdot \mathbf{r}}\left\langle e^{i\left(\phi_{\mathbf{q}_{1}}+\phi_{\mathbf{q}_{2}}\right)}\right\rangle \\
= & \sum_{\mathbf{q}} \frac{v^{2} s_{0}^{2}}{\left(\kappa q^{4}+\gamma q^{2}+v\right)^{2}} e^{i \mathbf{q} \cdot \mathbf{r}},
\end{aligned}
$$

which is exactly equal to Eq. 177.

Equation (178) needs to be adapted to a strip geometry. First, it is clear that, in rectangular coordinates,

$h_{c}(x, y)=\sum_{q_{x}} \sum_{q_{y} \geq 0} \frac{2 v s_{0}}{\kappa q^{4}+\gamma q^{2}+v} \cos \left(x q_{x}+y q_{y}+\phi_{q_{x}, q_{y}}\right)$.

Moreover, we can assume $q_{x}=2 \pi n_{x} / N_{x}$ and $q_{y}=2 \pi n_{y} / N_{y}$, with $n_{x}=-N_{x} / 2, \ldots,\left(N_{x}-1\right) / 2$ and $n_{y}=-N_{y} / 2, \ldots,\left(N_{y}-\right.$ 1) $/ 2$, where $N_{x} \times N_{y}$ is the number of grid points in real space. One could write the real space grid using the primitive lattice vectors of the hexagonal (actually triangular) underlying system. However, since the strip has a rectangular geometry and the field $h(x, y)$ is defined over a coarse grained lattice (hydrodynamic continuum limit) which does not need to reflect the underlying atomic structure. Thus, a rectangular mesh suffices and we can rewrite Eq. (179) as

$$
\begin{aligned}
h_{c}(x, y)= & \sum_{n_{x}=-\frac{N_{x}}{2}}^{\frac{N_{x}-1}{2}} \sum_{n_{y}=0}^{\frac{N_{y}-1}{2}} \frac{2 v s_{0}}{\kappa q^{4}+\gamma q^{2}+v} \\
& \quad \times \cos \left[2 \pi\left(x \frac{n_{x}}{N_{x}}+y \frac{n_{y}}{N_{y}}\right)+\phi_{n_{x}, n_{y}}\right],
\end{aligned}
$$

with $q^{2}=(2 \pi)^{2}\left[\left(n_{x} / N_{x}\right)^{2}+\left(n_{y} / N_{y}\right)^{2}\right]$, where we have implicitly assumed $N_{y}$ to be odd. 


\subsection{Vector potential (off-diagonal) disorder}

The off-diagonal disorder discussed in the previous Sections can be cast in terms of a random vector potential, as nicely reviewed in Ref. 67]. Here we briefly present the mapping of the tight-binding hopping disorder $\delta t_{i j}$ into a random vector potential $\mathbf{A}(x, y)$. We then show how to implement this kind of disorder in the tight-binding model.

The continuum limit of the honeycomb lattice tight-binding model near the neutrality points translates into a Dirac equation. Using the Bloch states of one (A or B) of the triangular sublattices (i.e., the underlying Bravais lattice) that constitute honeycomb lattice, one obtains the Hamiltonian

$\hat{H}_{\mathrm{AB}}=t\left(\begin{array}{cc}0 & 1+e^{i \mathbf{k} \cdot \mathbf{a}_{1}}+e^{i \mathbf{k} \cdot \mathbf{a}_{2}} \\ 1+e^{-i \mathbf{k} \cdot \mathbf{a}_{1}}+e^{-i \mathbf{k} \cdot \mathbf{a}_{2}} & 0\end{array}\right)$,

where $\mathbf{a}_{1}=\left(a_{0} / 2, a_{0} \sqrt{3} / 2\right)$ and $\mathbf{a}_{2}=\left(-a_{0} / 2, a_{0} \sqrt{3} / 2\right)$ are the primitive (Bravais) lattice vectors. Here we use the lattice vector conventions of Ref. [1]. The Hamiltonian $H$ acts on a spinor whose components are the envelop wave function amplitudes at the sublattices $\mathrm{A}$ and $\mathrm{B}$. The corresponding low-energy dispersion relation shows two inequivalent cones (valleys) centered at the $k$-space points

$\mathbf{K}=\left(-\frac{4 \pi}{3 a_{0}}, 0\right) \quad$ and $\quad \mathbf{K}^{\prime}=\left(\frac{4 \pi}{3 a_{0}}, 0\right)$.

Expanding $\mathbf{k}$ around these points, one obtains the Hamiltonians

$$
\begin{aligned}
& \hat{H}_{K}=v \hbar\left(\begin{array}{cc}
0 & k_{x}-i k_{y} \\
k_{x}+i k_{y} & 0
\end{array}\right)=v \hbar\left(k_{x} \hat{\sigma}_{x}+k_{y} \hat{\sigma}_{y}\right) \\
& \hat{H}_{K^{\prime}}=v \hbar\left(\begin{array}{cc}
0 & -k_{x}-i k_{y} \\
-k_{x}+i k_{y} & 0
\end{array}\right)=v \hbar\left(-k_{x} \hat{\sigma}_{x}+k_{y} \hat{\sigma}_{y}\right),
\end{aligned}
$$

where $k_{x}$ and $k_{y}$ are measured from the cone vertices. The real-space, continuum version of the Hamiltonians can be obtained by replacing $k_{x}$ with $-i \partial_{x}$ and $k_{y}$ with $-i \partial_{y}$. As a result,

$$
\begin{aligned}
\hat{H}_{K} & =v \hbar\left(\begin{array}{cc}
0 & -i \partial_{x}-\partial_{y} \\
-i \partial_{x}+\partial_{y} & 0
\end{array}\right) \\
\hat{H}_{K^{\prime}} & =v \hbar\left(\begin{array}{cc}
0 & i \partial_{x}-\partial_{y} \\
i \partial_{x}+\partial_{y} & 0
\end{array}\right) .
\end{aligned}
$$

When a vector potential is present, the substitution is instead $k_{x, y} \longrightarrow-i \partial_{x, y}+\frac{e}{\hbar c} A_{x, y}$ (with $e>0$ ) and the Hamiltonians become

$$
\hat{H}_{K}=v \hbar\left(\begin{array}{cc}
0 & -i \partial_{x}-\partial_{y}+\frac{e}{\hbar c}\left(A_{x}-i A_{y}\right) \\
-i \partial_{x}+\partial_{y}+\frac{e}{\hbar c}\left(A_{x}+i A_{y}\right) & 0
\end{array}\right)
$$

$$
\hat{H}_{K^{\prime}}=v \hbar\left(\begin{array}{cc}
0 & i \partial_{x}-\partial_{y}-\frac{e}{\hbar c}\left(A_{x}+i A_{y}\right) \\
i \partial_{x}+\partial_{y}-\frac{e}{\hbar c}\left(A_{x}-i A_{y}\right) & 0
\end{array}\right) .
$$

We now show how a local (long-ranged) distortion in the lattice gives raise to $H_{K\left(K^{\prime}\right)}$ as above. Let us assume that the three nearest-neighbor hopping amplitudes of any given site are not equal: Calling them $t_{0}, t_{1}$, and $t_{2}$, we have to rewrite Eq. (182) in the form

$\hat{H}_{\mathrm{AB}}=\left(\begin{array}{cc}0 & t_{0}+t_{1} e^{i \mathbf{k} \cdot \mathbf{a}_{1}}+t_{2} e^{i \mathbf{k} \cdot \mathbf{a}_{2}} \\ t_{0}+t_{1} e^{-i \mathbf{k} \cdot \mathbf{a}_{1}}+t_{2} e^{-i \mathbf{k} \cdot \mathbf{a}_{2}} & 0\end{array}\right)$.

Expanding around the same $\mathbf{K}$ and $\mathbf{K}^{\prime}$ points, and assuming $\left|t_{1}-t_{2}\right| \ll\left|t_{1}+t_{2}\right|$, we find

$$
\begin{aligned}
\hat{H}_{K} & =v \hbar\left(\begin{array}{cc}
0 & k_{x}-i k_{y} \\
k_{x}+i k_{y} & 0
\end{array}\right) \\
& +\left(\begin{array}{cc}
0 & t_{0}-\frac{t_{1}+t_{2}}{2}-\frac{i \sqrt{3}\left(t_{1}-t_{2}\right)}{2} \\
t_{0}-\frac{t_{1}+t_{2}}{2}+\frac{i \sqrt{3}\left(t_{1}-t_{2}\right)}{2} & 0
\end{array}\right) \\
\hat{H}_{K^{\prime}} & =v \hbar\left(\begin{array}{cc}
0 & -k_{x}-i k_{y} \\
-k_{x}+i k_{y} & 0
\end{array}\right) \\
& +\left(\begin{array}{cc}
0 & t_{0}-\frac{t_{1}+t_{2}}{2}+\frac{i \sqrt{3}\left(t_{1}-t_{2}\right)}{2} \\
t_{0}-\frac{t_{1}+t_{2}}{2}-\frac{i \sqrt{3}\left(t_{1}-t_{2}\right)}{2} & 0
\end{array}\right) .
\end{aligned}
$$

Thus, we can define two vector potentials, one for each cone:

$A_{x}^{K}=\frac{c}{v e}\left(t_{0}-\frac{t_{1}+t_{2}}{2}\right), \quad A_{y}^{K}=\frac{c}{v e} \frac{\sqrt{3}}{2}\left(t_{1}-t_{2}\right)$,

and

$A_{x}^{K^{\prime}}=-\frac{c}{v e}\left(t_{0}-\frac{t_{1}+t_{2}}{2}\right), \quad A_{y}^{K^{\prime}}=-\frac{c}{v e} \frac{\sqrt{3}}{2}\left(t_{1}-t_{2}\right)$.

Notice that $\mathbf{A}^{K}=-\mathbf{A}^{K^{\prime}}$, as expected from time-reversal symmetry considerations.

We can use the vector potential as a gauge field that parameterizes local fluctuations in the nearest-neighbor hopping matrix elements. For this purpose, it is useful make a single-cone approximation and rewrite Eq. 193 in the form

$t_{0}(n, j)=t+\frac{2 v e}{c} A_{x}(n, j)$,

$t_{1}(n, j)=t+\frac{v e}{c}\left[A_{x}(n, j)+\frac{1}{\sqrt{3}} A_{y}(n, j)\right]$,

$t_{2}(n, j)=t+\frac{v e}{c}\left[A_{x}(n, j)-\frac{1}{\sqrt{3}} A_{y}(n, j)\right]$,

where $(n, j)$ are the coordinates of sites belonging to one of the sublattices. Notice that this type of off-diagonal disorder does break particle-hole symmetry.

Another way to proceed and get the same results is to use a Peierls substitution in the hopping matrix elements [68], such that

$e^{i \mathbf{k} \cdot \mathbf{a}_{1}} \longrightarrow e^{i\left(\mathbf{k}+\frac{e}{\hbar c} \mathbf{A}\right) \cdot \mathbf{a}_{1}}$

$e^{i \mathbf{k} \cdot \mathbf{a}_{2}} \longrightarrow e^{i\left(\mathbf{k}+\frac{e}{\hbar c} \mathbf{A}\right) \cdot \mathbf{a}_{2}}$ 
in Eq. 182. For instance, expanding on $\mathbf{A}$ and around the K point, we obtain

$$
\begin{aligned}
t\left[1+e^{i\left(\mathbf{k}+\frac{e}{\hbar c} \mathbf{A}\right) \cdot \mathbf{a}_{1}}+e^{i\left(\mathbf{k}+\frac{e}{\hbar c} \mathbf{A}\right) \cdot \mathbf{a}_{2}}\right] \approx \\
\hbar v\left(k_{x}-i k_{y}\right)+\frac{e v}{c}\left(A_{x}-i A_{y}\right) .
\end{aligned}
$$

Then, setting

$t_{0}-\frac{t_{1}+t_{2}}{2}-i \sqrt{3} \frac{t_{1}-t_{2}}{2}=\frac{e v}{c}\left(A_{x}-i A_{y}\right)$,

we arrive at Eqs. (195) to 197.

This concludes the demonstration that a hopping distortion can be mapped into a corresponding vector field. From the point of view of a tight-binding modeling and the RGF method, it seems simpler to model strain with renormalized hoppings, thus avoiding issues related to projections onto the $\mathbf{K}$ and $\mathbf{K}^{\prime}$ cones to preserve time-reversal symmetry.

The random gauge potential model is also interesting for other reasons. It has a physical realization in rippled graphene subjected to a strong parallel magnetic field [69] and has been analytically addressed by several authors, e.g. Ref. [4].

Let us model the random vector potential by assuming that the gauge field has Gaussian fluctuations, such that

$$
\left\langle A_{\alpha}(n, j) A_{\beta}\left(n^{\prime}, j^{\prime}\right)\right\rangle=\lambda \delta_{\alpha \beta} e^{-\left|\mathbf{r}_{n, j}-\mathbf{r}_{n^{\prime}, j^{\prime}}\right|^{2} / 2 \xi^{2}},
$$

where $\lambda$ measures the strength of the fluctuations and $\xi$ is their correlation length. One way to generate this correlation function is to define at the nodes of a regular lattice of constant $a_{g}$ two sets of uniformly distributed random number $3\left\{c_{k}^{\alpha}\right\}_{k=1, \ldots, N}$ and to define the gauge field through the expression

$A_{\alpha}(n, j)=\frac{f}{\mathscr{C}} \sum_{k=1}^{\mathscr{N}} c_{k}^{\alpha} e^{-\left|\mathbf{r}_{n, j}-\mathbf{R}_{k}\right|^{2} / \xi^{2}}$,

where

$\mathscr{C}=\sum_{k=1}^{\mathscr{N}} e^{-\left|\mathbf{r}_{n, j}-\mathbf{R}_{k}\right|^{2} / \xi 2} \longrightarrow \pi\left(\frac{\xi}{a_{g}}\right)^{2}$

and $\lambda=f^{2}\left(a_{g} / \xi\right)^{2} / 2 \pi$ when $\mathscr{N} \rightarrow \infty$ maintaining $\mathscr{N} a_{g}<$ $\infty$. This construction implicitly assumes that $a_{g} \ll \xi$.

There is a useful way to quantify the fluctuations of the vector potential. Let us denote $\left\langle\Phi^{2}\right\rangle$ the rms value of the magnetic flux piercing a region of area $\mathscr{A}<\xi^{2}$. Then, $\left\langle\Phi^{2}\right\rangle \approx$ $\mathscr{A}^{2}\left\langle B^{2}\right\rangle$, where $B=\partial_{x} A_{y}-\partial_{y} A_{x}$. Following the steps shown in Appendix $C$, we find that $\left\langle B^{2}\right\rangle=f^{2}\left(a_{g} / \xi\right)^{2} / 2 \pi \xi^{2}$.

We can now redefine the vector potential to absorb the prefactor $e / \hbar c$ : $\tilde{\mathbf{A}} \equiv(e / \hbar c) \mathbf{A}$. Likewise, in order to get rid of the prefactor in the expressions used to generate the vector potential, we introduce $\tilde{f} \equiv(\hbar c / e) f$ and $\tilde{\lambda} \equiv(\hbar c / e)^{2} \lambda$.

\footnotetext{
3 One set of random numbers for each $\alpha$-component of the gauge field, such that $\left\langle c_{k}^{\alpha}\right\rangle=0$ and $\left\langle c_{k}^{\alpha} c_{k^{\prime}}^{\alpha^{\prime}}\right\rangle=\delta_{k k^{\prime}} \delta_{\alpha \alpha^{\prime}}$.
}

Then, we can write the following expression for the estimated rms value of the random magnetic flux in units of the flux quantum $\left(\Phi_{0}=h c / e\right)$ :

$\frac{\delta \varphi}{2 \pi} \equiv \frac{\sqrt{\left\langle\Phi^{2}\right\rangle}}{\Phi_{0}} \approx \frac{1}{2 \pi} \frac{1}{\sqrt{2 \pi}}\left(\frac{\mathscr{A}}{\xi^{2}}\right) a_{g} \tilde{f}$,

which implies $\tilde{\lambda}=(\delta \varphi)^{2} \xi^{2} / \mathscr{A}^{2}$. Thus, the relation between the rms flux phase piercing an elementary hexagon $\mathscr{A}_{\mathrm{hex}}=$ $\sqrt{3} a_{0}^{2} / 2$ and the vector potential intensity is

$\tilde{f}_{\text {hex }}=\sqrt{\frac{8 \pi}{3}}\left(\frac{\xi}{a_{0}}\right)^{2} \frac{\delta \varphi}{a_{g}}$,

or, equivalently,

$\frac{\tilde{f}_{\text {hex }}}{\mathscr{C}}=\sqrt{\frac{8}{3 \pi}}\left(\frac{a_{g}}{a_{0}}\right)^{2} \frac{\delta \varphi}{a_{g}}$.

On the other hand, if we set $\mathscr{A}_{\text {ripple }}=\xi^{2}$ to denote the typical area of a ripple, we obtain

$\tilde{f}_{\text {ripple }}=\sqrt{2 \pi} \frac{\delta \varphi}{a_{g}}, \quad \frac{\tilde{f}_{\text {hex }}}{\mathscr{C}}=\sqrt{\frac{2}{\pi}}\left(\frac{a_{g}}{\xi}\right)^{2} \frac{\delta \varphi}{a_{g}}$.

\subsection{Edge disorder}

Etching a graphene sheet to produce nanoribbons always leaves behind some roughness at the edges. When the irregular shape of the boundaries of the propagating region is very pronounced, it leads to the formation of "bottlenecks" and "cavities", which tend to increase charging effects and lead to Coulomb blockade oscillations of the conductance [70]. However, even mild amounts of edge disorder can affect dramatically electronic transport in nanoribbons. In this case, it has been proposed that for long enough nanoribbons, Anderson localization (and thus an insulating behavior) can develop [71,72,73]. Insulating behavior, albeit of a different nature, is also expected in "perfect" nanoribbons due to lattice symmetric breaking caused by the deformation of the chemical bonds involving carbon atoms at the edges, as revealed by DFT calculations [45]

Edge disorder can be simulated by considering slices with random numbers sites (see Fig. 14): For instance, we can draw the number of sites $M_{n}$ of the $n$th slice randomly according to the Gaussian distribution

$P\left(M_{n}\right)=\frac{1}{\sqrt{2 \pi} \delta M} e^{-\left(M_{n}-\bar{M}\right)^{2} / 2 \delta M^{2}}$

where $\bar{M}$ is the average number of transverse unit cells in the nanoribbon and $\delta M$ is its standard deviation. Other distributions can be investigated straightforwardly. The slices are concatenated such that hopping matrix elements connecting sites which fall into empty spaces are set to zero (although 
this can be avoided when programming the recursive calculation by using nested loops with variable ranges). Thus, one may think of this procedure as a random removal of sites at the edges of the nanoribbon. This approach has been used to study the existence of localized states in graphene systems [71].

Another approach, which tries to mimic the effect of etching, is explained in [73]. Again, the numbers $\left\{M_{n}\right\}$ are considered random variables, but their generation follows a different procedure. One visits sequentially each edge site (at the top and bottom) and elects to removes it (or not) according to a probability $p_{1}$. Certain sites, when removed, require the removal of neighboring sites as well, as an edge configuration where carbon atoms have a single bond are not stable (unless both dangling bonds in the carbon atom are pacified, but this is not likely to occur during etching). After this first sweep of edge sites, a second sweep follows, but now sites are removed with a probability $p_{2}$. One can continue repeating this procedure, using a different removal probability at each sweep, until the desired amount of roughness is obtained.

\section{Some Numerical Results}

In this Section we show some representative results obtained with the RGF method. We begin by addressing the case of ballistic transport in graphene sheets where analytical results are known and serve to benchmark the numerical method. Next, we discuss the case of graphene sheets with longrange disorder, where the RGF method was used to clarify the controversial issue of the "universal conductivity minimum". Finally, we present results for graphene nanoribbons, showing how the method can be used to calculate LDOS and the local current density.

\subsection{Ballistic transport in clean samples}

Here we present results obtained for the case of ballistic transport in graphene sheets. We consider mainly the armchair orientation, since this, in the clean limit, provides a band structure and dispersion relation very similar to a quasione-dimensional projection of the Dirac fermion model and is more suitable for scaling analyzes.

First, in Fig. 10, we show results for the clean limit (no bulk or edge disorder) for a short ribbon, keeping the back gate voltage fixed to zero (neutrality point) and varying the Fermi energy in the contacts (zero bias). The numerical data is compared to the analytical expressions derived by in Ref. [39]. The agreement is quite good for large systems and becomes worse when the system is too small (not shown). In particular, a strong asymmetry and a lack of well-defined
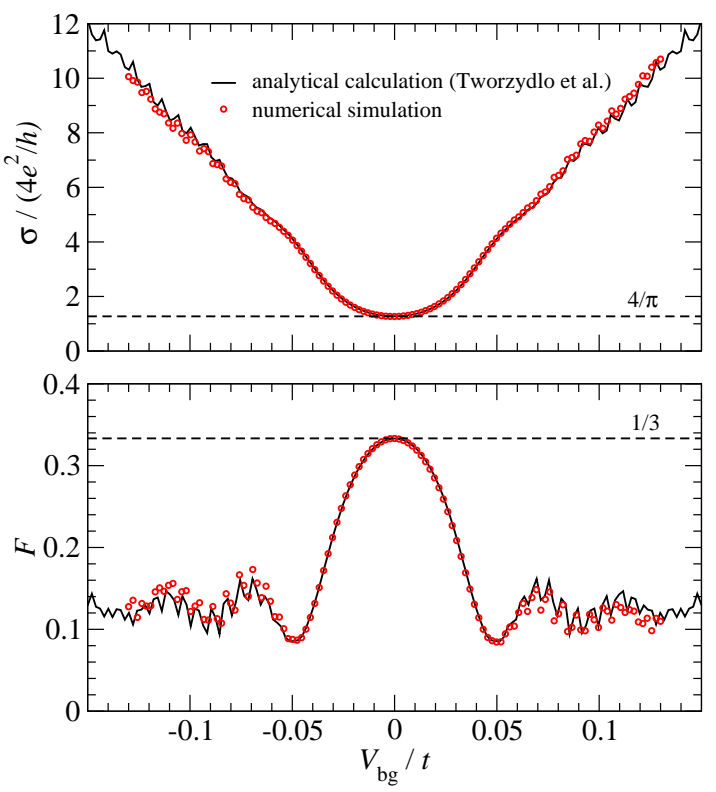

Fig. 10 Results of linear transport calculations for clean graphene sheets: Conductivity (defined as $\sigma=L G / W$ ) and Fano factor as a function of the Fermi energy in the contacts $\left(V_{g}=0\right)$. The band in the square lattice leads was offset such that its middle coincides with the neutrality point in the graphene sheet in order to increase the density of states at the contact and thus mimic a metallic lead. Armchair edges, $M=360$ and $N=70$ (aspect ratio $W / L=5.2$ ).

oscillations occurs if the system is not large enough (not shown).

The ability of the recursive method to get precise results for clean systems is clear also in Fig. 11, where the calculations are performed for different aspect ratios. The deviations from the analytical curve only occur when the system is too short and evanescent modes dominate transport.

In Figs. 12 we show the conductance and the current density in the linear regime for small flakes with armchair and zigzag edges. Here, the leads are also honeycomb lattices. In this case, the conductance steps can be easily understood from the energy dispersion relation of graphene infinite ribbons [74], namely, the dimensionless conductance $G /\left(2 e^{2} / h\right)$ is given by the number of bands crossing the Fermi energy $E$.

There is no such simple explanation for the current density. Notice that the notable difference in the current distribution for armchair and zigzag orientations at $E=0$ : While in the latter the current is primarily carried by edge states, in the former the current is uniformly distributed across the flake. As one moves a just little bit away from $E=0$, the current distribution for the zigzag flake changes drastically, with nearly no current running at the edges. This result is related to the the fact that for zigzag nanoribbons the $E=0$ states are strongly localized at the edges. As soon as $|E|>0$ both edge states and edge currents disappear, even in the case of a single conducting channel. For the armchair orien- 

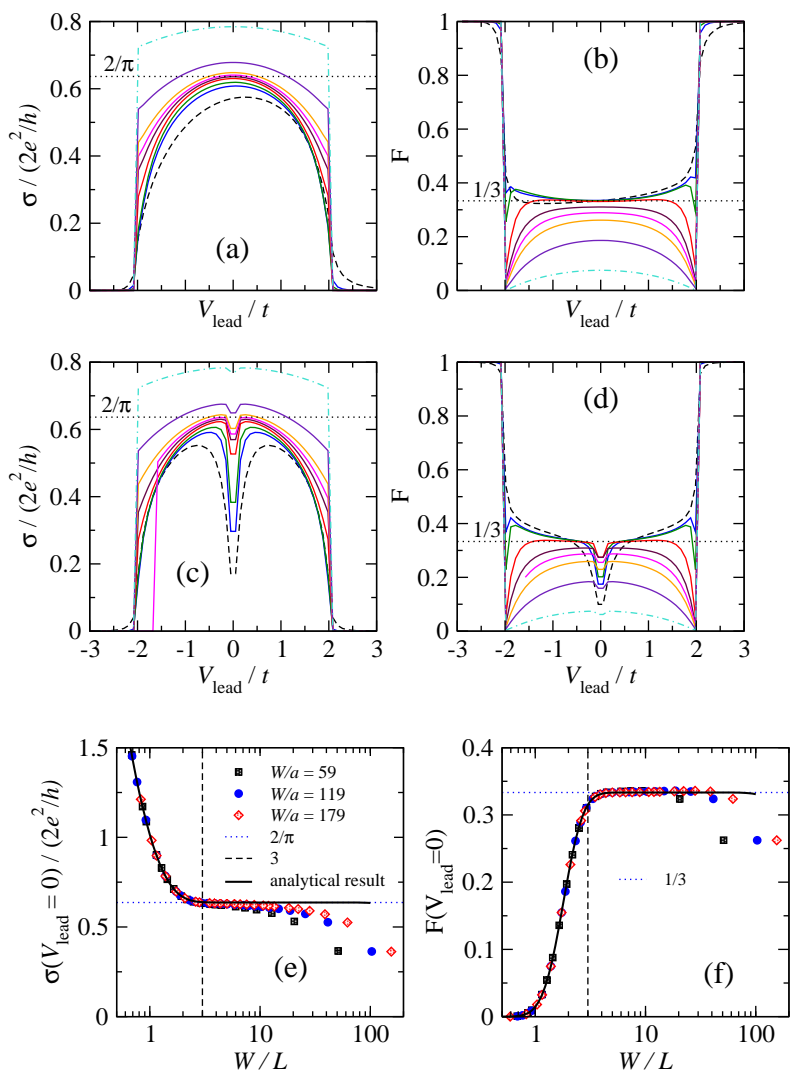

Fig. 11 Results of numerical simulations of clean ribbons: conductivity and Fano factor as a function of the Fermi energy in the contacts $\left(V_{g}=0\right)$ for different aspect ratios. (a), (b), (e), (f): Square lattice contacts; (c) and (d): Armchair honeycomb contacts. For all plots, $M=120$. In plots (a), (b), (c), and (d), the value of $N$ are 12 (dashed line), 24, 36, 72, 96, 108, 120, 148, and 200 (dashed-dotted line). The thick solid line corresponds to the analytical result [39].

tation, the change in the current distribution for increasing energies is less drastic.

It should be stressed the edge current densities of zigzag nanoribbons change both quantitative and qualitative if one switches from nearest-neighbor [75] to next-nearest-neighbor tight-binding models [76]. The issue of which model is appropriate is tied to the desire to fit DFT calculations [45] or to explain experimental manipulation and characterization of nanoribbon edges [77].

\subsection{Disordered graphene sheets}

The conductivity minimum $\sigma_{0}$ observed at the charge neutrality point in graphene monolayers has been a subject of intense debate, which is reviewed, for instance, in Ref. [2]. Here, we show how the long-range Gaussian correlated potential can be used to investigate the value of $\sigma_{0}$. 4

\footnotetext{
${ }^{4}$ The discussion and results that follow complement the material presented in Ref. [2].
}

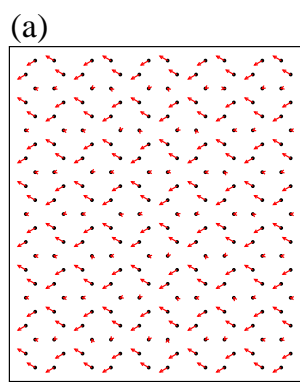

(b)
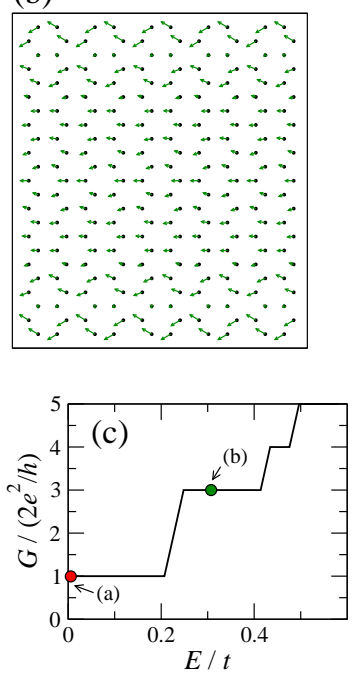

(d)

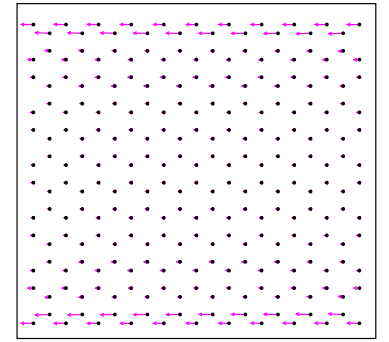

(e)
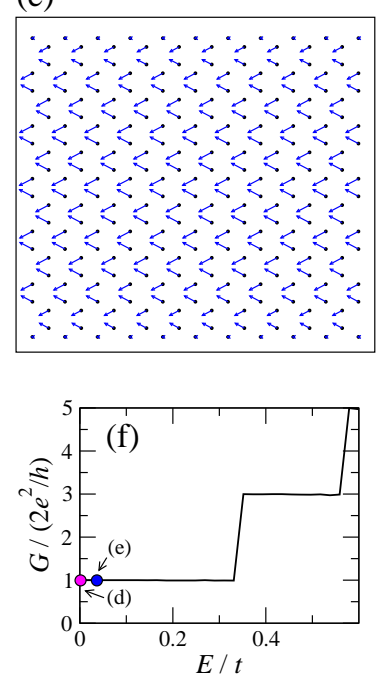

Fig. 12 Current densities (a,b,d,e) and linear conductance steps (c,d) of two small ballistic graphene ribbons. In (a,b,c,d) the arrows represent the current densities (in arbitrary units) evaluated at different sites using Eq. (112). Armchair edges, $M=12, N=20$ : (a), (b), and (c); zigzag edges, $M=12, N=21$ : (d), (e), and (f). Energies: $E=0$ for plots (a) and (d); $E=0.3 t$ for plot (b); $E=0.01 t$ for plot (e). amplitude.

In the diffusive regime, in general, the system geometry has little influence on the transport properties which allows one to express the average conductivity as $\sigma=(L / W)\langle G\rangle$, where $L$ is the system length and $W$ its width. We use the same setting as in the previous subsection, including now long-range Gaussian disorder in the device region. To generate the data shown in Fig. 13 four different aspect ratios were considered as well as several values of $K_{0}$ and $\xi / a$. The average conductivity $\sigma_{0}$ obtained from $\left\langle G\left(V_{g}=0\right)\right\rangle$ is plotted versus $L$ scaled by $\ell^{*}$. The parameter $\ell^{*}$ depends on $K_{0}$ and $\xi$. We identify $\ell^{*}$ with the elastic disorder mean free path $\ell$.

Let us summarize the results shown in Fig.13. Two clear regimes can be identified. For $L / \ell \ll 1$, the probability of an electron being scattered by disorder as it traverses the sample is very small. This corresponds to the ballistic regime, where scattering occurs mainly at the sample edges and transport properties are dominated by the sample geometry. Note that when $L / \ell<1, \sigma_{0}$ approaches the prediction for the pure ballistic case [39], indicated by the arrows in Fig. 13 In con- 


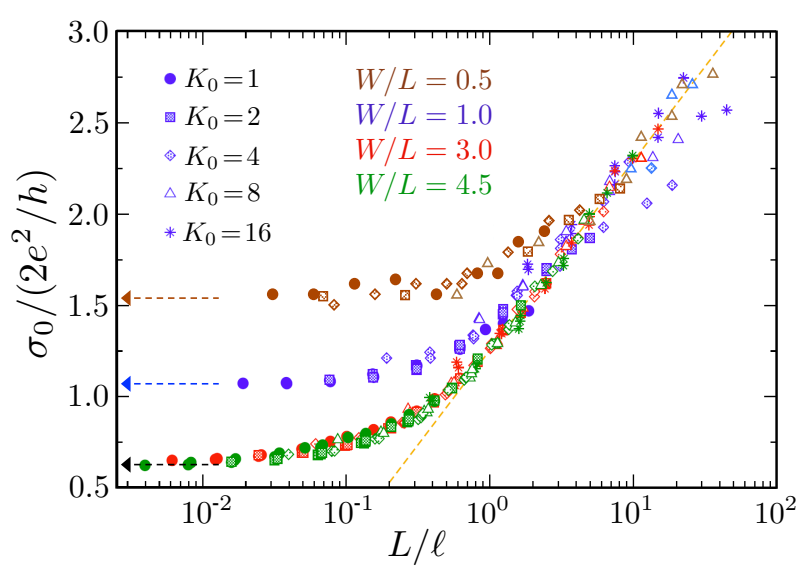

Fig. 13 Conductivity minimum $\sigma_{0}$ in unit of $2 e^{2} / h$ as a function of the system size $L$ scaled by the electron mean free path $\ell$. The results correspond to the average over $10^{2} \cdots 10^{4}$ disorder realizations for $L$ ranging between 50 and $500 a_{0}$. The colors represent different aspect ratios $W / L$. The symbols stand for the values of the dimensionless disorder strength $K_{0}$. The arrows indicate the analytical value of the conductivity minimum in the ballistic limit [39], which depends on $W / L$. The dotted line gives the diffusive $\ln (L / \ell)$ behavior [4].

trast, when $L / \ell \gg 1$, the system becomes diffusive and geometry affects transport weakly. Figure 13 clearly shows this crossover. For the diffusive regime, $L / l \gg 1$, the conductivity is proportional to $\ln (L / \ell)$, in agreement with the nonlinear sigma model prediction [4]. The mismatch between the numerical prefactor for the logarithm and the value characteristic of the symplectic class may be related to the finite contact resistance [62] present in our simulations.

These simulations suggest an explanation for results obtained in transport experiments at the charge neutrality point. In the coherent diffusive regime, the conductivity minimum has significant sample-to-sample fluctuations and its average shows a weak (logarithmic) dependence on the mean free path. Typical diffusive experimental samples have $L / \ell \approx$ $1-10$ and $\sigma_{0} \approx 4 e^{2} / h$, similarly to what is shown in Fig. 13 .

\subsection{Nanoribbons}

For nanoribbons, both bulk and edge disorder play a role in electronic transport. In the absence of band gaps, longrange disorder does not suppress conductance significantly and a perfect conducting channel exists near the neutrality point [78, 79, 80]. The story is quite different for short-range disorder. Bulk imperfections (lattice defects, impurities, or adsorbates) and edge imperfections can lead to strong localization due to backscattering and enhanced destructive interference [72,73]. To illustrate this point, Fig. 14 shows the rapid smearing of the linear conductance steps of a nanoribbon when even a small amount of edge sites are randomly removed (i.e., etched out). This shows how challenging it is to observe conductance quantization experimentally in these systems.
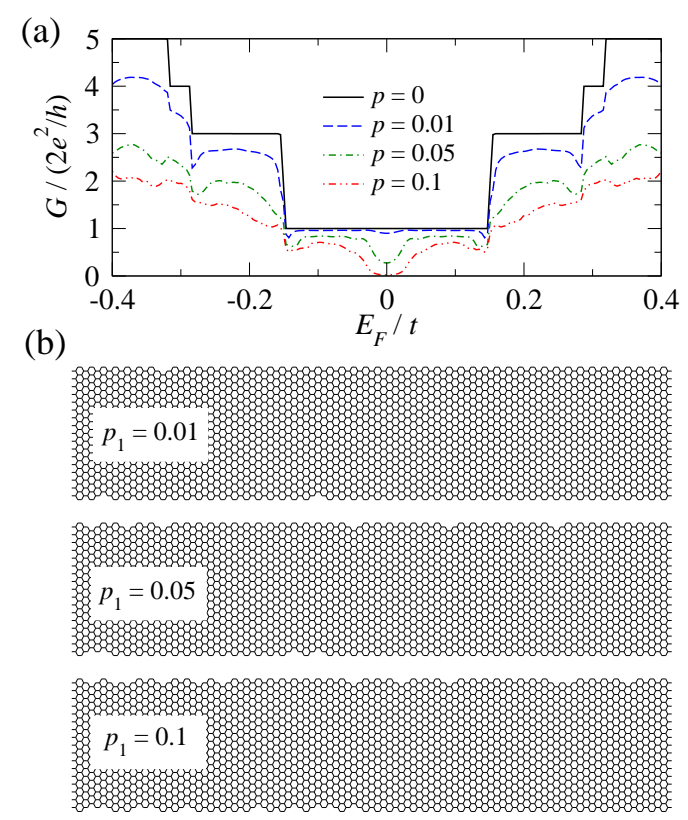

Fig. 14 (a) Linear conductance of edge disordered nanoribbons with armchair edge orientation, $M=18, N=200$, averaged over 100 realizations, as a function of energy. Only one etching sweep is used, but results for three different values of the site removal probability $p_{1}$ are shown. (b) Typical realizations used in (a) for value of $p_{1}$ considered.

The appearance of localized states in nanoribbons with edge disorder is demonstrated in Fig. 15 where the linear conductance and the local density of states for a nanoribbon are shown in the cases of perfect and irregular edges.

Acknowledgements Financial support by the Brazilian funding agencies FAPERJ and CNPq is gratefully acknowledged.

\section{A Steps in the linear conductance}

Let us show that the surface Green's function in Eq. 67] leads to the expect steps in the linear conductance. For this purpose, let us begin by noticing that, in the case of a square lattice lead, only propagating modes yield a finite level width: For $\left|E-\varepsilon_{v}\right|<2 t_{x}$,

$\tilde{\Gamma}_{v}=-2 \operatorname{Im}\left[\tilde{\Sigma}_{v}\right]=2 \operatorname{Im}\left[\left(\tilde{g}_{v}\right)^{-1}\right]=2 t_{x} \sin \phi_{v}$

where $\sin \phi_{V}=\sqrt{1-\left(E-\varepsilon_{V}\right)^{2} / 4 t^{2}}$, in which case we can write $\tilde{g}_{v}=$ $e^{-i \phi_{v}} / t_{x}$.

In order to obtain the retarded Green's function across the system, we add one slice between the left and right contacts and use the following expression, easily derivable from Eqs. 32, , 33, , 41, and 422:

$G_{0,2}=t_{x}^{2} g_{L}^{2}\left(g_{L}^{-1}-t_{x}^{2} g_{L}\right)^{-1}$

Since $G_{0,2}$ depends solely on $g_{L}$, we can rewrite in the propagation mode basis, in which case the Landauer formula is reduced to [see Eq. [15]

$\mathscr{T}=\sum_{v}^{\prime} \tilde{\Gamma}_{v}\left(\tilde{G}_{0,2}\right)_{v} \tilde{\Gamma}_{v}\left(\tilde{G}_{0,2}\right)_{v}^{*}$, 


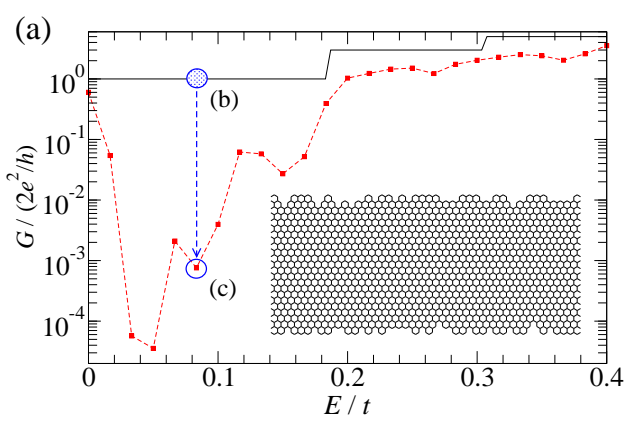

(b)

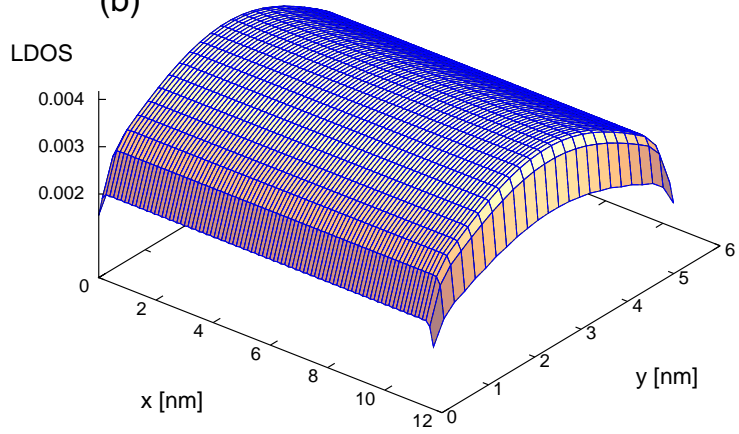

(c)

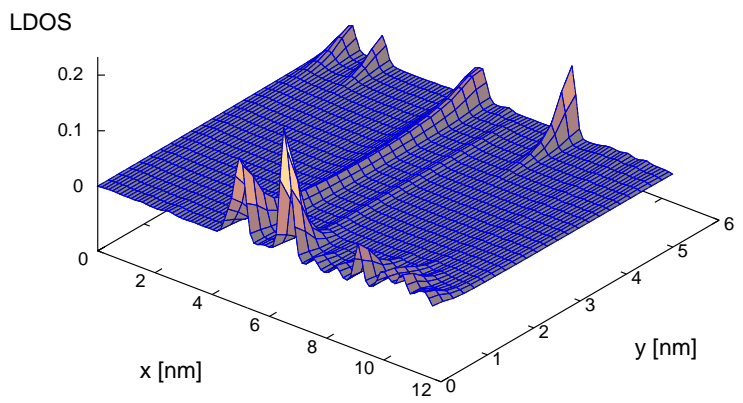

Fig. 15 (a) The linear conductance of a short nanoribbon (zigzag edges, $M=24, N=91$ sites) as a function of energy. The solid line corresponds to perfect edges while the dashed line corresponds to the edge disorder realization shown in the inset (three etching sweeps with $p_{1}=0.3, p_{2}=0.2, p_{3}=0.1$ ). Surface plot of the local density of states (arbitrary units, brickwall lattice representation) for the same nanoribbon at the energy value highlighted in (a): (b) clean case; (c) edge disordered case. Notice the appearance of localized states that traverse the nanoribbon when edge disorder is present. (The actual LDOS was convoluted with a Gaussian profile to smooth out high-frequency oscillations.)

where the prime indicates that the sum runs only over states such that $\left|E-\varepsilon_{v}\right|<2 t_{x}$ and

$$
\left(\tilde{G}_{0,2}\right)_{v}=t_{x}^{2} \tilde{g}_{v}^{2}\left(\tilde{g}_{v}^{-1}-t_{x}^{2} \tilde{g}_{v}\right)^{-1}=\frac{e^{-2 i \phi_{v}}}{2 i t_{x} \sin \phi_{v}} .
$$

Putting all together, we find that

$$
\begin{aligned}
\mathscr{T} & =\sum_{v}^{\prime} \tilde{\Gamma}_{v}^{2}\left|\left(\tilde{G}_{0,2}\right)_{v}\right|^{2} \\
& =\sum_{v}^{\prime} 1=\# \text { propagating modes for a given } E,
\end{aligned}
$$

which is the expected result for a clean ballistic system.

\section{B Peierls hopping phases}

Here we evaluate the phase of the hopping matrix elements between any two arbitrary sites due to the presence of a perpendicular magnetic field. We pick the vector potential in the generic Landau gauge $A_{x}=$ $(\alpha-1) B y$ and $A_{y}=\alpha B x$, with $0 \leq \alpha \leq 1$. The (directional) Peierls phase between two neighboring sites $k$ and $k^{\prime}$ is given by [68]

$$
\begin{aligned}
\varphi_{k, k^{\prime}}= & \frac{e c}{\hbar} \int_{k}^{k^{\prime}} \mathbf{A} \cdot d \mathbf{l} \\
= & \frac{e c B}{\hbar}\left[(1-\alpha) \cos \theta_{k k^{\prime}} \int_{y_{k}}^{y_{k^{\prime}}} y d l+\alpha \sin \theta_{k k^{\prime}} \int_{x_{k}}^{x_{k^{\prime}}} x d l\right] \\
= & \frac{e c B}{\hbar}\left[(1-\alpha) \operatorname{cotan} \theta_{k k^{\prime}} \int_{y_{k}}^{y_{k^{\prime}}} y d y+\alpha \tan \theta_{k k^{\prime}} \int_{x_{k}}^{x_{k^{\prime}}} x d x\right] \\
=\frac{e c B}{\hbar} & {\left[(1-\alpha)\left(x_{k^{\prime}}-x_{k}\right)\left(\frac{y_{k^{\prime}}+y_{k}}{2}\right)\right.} \\
& \left.\quad+\alpha\left(y_{k^{\prime}}-y_{k}\right)\left(\frac{x_{k^{\prime}}+x_{k}}{2}\right)\right],
\end{aligned}
$$

where $\theta_{k k^{\prime}}$ is the angle that the segment $k-k^{\prime}$ makes with the $x$ axis.

Notice that $\varphi_{k, k^{\prime}}=-\varphi_{k^{\prime}, k}$. If we sum over all the bond phases around the perimeter of a hexagon, we obtain $\sum \varphi=\sqrt{3} e c B a_{0}^{2} / 2 \hbar=$ $2 \pi\left(\Phi / \Phi_{0}\right)$, where $\Phi_{0}=h / e c$ (flux quantum), and $\Phi=B A_{\text {hex }}$, with $A_{\text {hex }}=\sqrt{3} a_{0}^{2} / 2$ being the area of the hexagon.

\section{Random Flux Estimate}

Let us estimate the rms value of the random magnetic field produced by the random vector potential:

$$
\begin{aligned}
\left\langle B^{2}\right\rangle & =\left\langle\left(\partial_{x} A_{y}-\partial_{y} A_{x}\right)^{2}\right\rangle \\
& =\left\langle\left\{\frac{f}{\mathscr{C}} \sum_{k}\left[c_{k}^{x} \frac{2\left(x_{n, j}-X_{k}\right)}{\xi^{2}}+c_{k}^{y} \frac{2\left(y_{n, j}-Y_{k}\right)}{\xi^{2}}\right] e^{-\left|\mathbf{r}_{n, j}-\mathbf{R}_{k}\right|^{2} / \xi^{2}}\right\}^{2}\right\rangle \\
& =\frac{4 f^{2}}{\mathscr{C}^{2} \xi^{4}} \sum_{k}\left|\mathbf{r}_{n, j}-\mathbf{R}_{k}\right|^{2} e^{-2\left|\mathbf{r}_{n, j}-\mathbf{R}_{k}\right|^{2} / \xi^{2}} \\
& \approx \frac{4 f^{2}}{\mathscr{C}^{2} \xi^{4} a_{g}^{2}} \int d^{2} R R^{2} e^{-2 R^{2} / \xi^{2}} \\
& \approx \frac{\pi f^{2}}{2 \mathscr{C}^{2} a_{g}^{2}}=\frac{f^{2} a_{g}^{2}}{2 \pi \xi^{4}} .
\end{aligned}
$$

\section{References}

1. Castro Neto, A. H. F. Guinea, F., Peres, N. M. R. , Novoselov, K. S., Geim, A. K.: The electronic properties of graphene. Rev. Mod. Phys. 81, 109 (2009)

2. Mucciolo, E.R., Lewenkopf, C.H.: Disorder and electronic transport in graphene. J. Phys.: Condens. Matter 22, 273201 (2010)

3. Shon N.H., Ando, T.: Quantum transport in two-dimensional graphite system. J. Phys. Soc. Jap. 67, 2421 (1998) 
4. Ostrovsky, P.M., Gornyi, I.V., Mirlin, A.D.; Electron transport in disordered graphene. Phys. Rev. B 74235443 (2006)

5. Nomura K., MacDonald, A. H.: Quantum transport of massless Dirac fermions. Phys. Rev. Lett. 98, 076602 (2007)

6. Nomura, K., Koshino, M., Ryu, S.: Topological delocalization of two-dimensional massless Dirac fermions. Phys. Rev. Lett. 99, 146806 (2007)

7. Tworzydło, J., Groth, C.W., Beenakker, C.W.J.: Finite difference method for transport properties of massless Dirac fermions. Phys. Rev. B 78, 235438 (2008)

8. Hernández, A.R., Lewenkopf, C.H.: Finite-difference method for transport of two-dimensional massless Dirac fermions in a ribbon geometry, Phys. Rev. B 86, 155439 (2012)

9. Thouless, D.J., Kirkpatrick, S.: Conductivity of the disordered linear chain. J. Phys. C 14, 235 (1981)

10. Drouvelis, P. S., Schmelcher, P., Bastian, P.: Parallel implementation of the recursive Green's function method. J. Comp. Phys. 215, 741 (2006)

11. MacKinnon, A: The calculation of transport properties and density of states of disordered solids. Z. Phys. B 59, 385 (1985)

12. Sols, F, Macucci, M., Ravaioli, U., Hess, K.: Theory for a quantum modulated transistor. J. Appl. Phys. 66, 3892 (1989)

13. Baranger, H.U., DiVincenzo, D.P., Jalabert, R.A., Stone, A.D.: Classical and quantum ballistic-transport anomalies in microjunctions. Phys. Rev. B 44, 10637 (1991)

14. Kazymyrenko, K., Waintal, X.: Knitting algorithm for calculating Green functions in quantum systems. Phys. Rev. B 77, 115119 (2008)

15. Kramer, T., Kreisbeck, C., Krueckl, V.: Wave-packet dynamics approach to transport in mesoscopic systems. Phys. Scr. 82, 038101 (2010)

16. Yuan, S., De Raedt, H., Katsnelson, M.I.: Modeling electronic structure and transport properties of graphene. with resonant scattering centers Phys. Rev. B 82115448 (2010)

17. Weiße, A., Wellein, G., Alvermann A., Fehske, H.: The kernel polynomial method. Rev. Mod. Phys. 78, 275 (2006)

18. Ferreira, A., Viana-Gomes, J., Nilsson, J., Mucciolo, E. R., Peres, N.M.R., Castro Neto, A.H.: Unified description of the dc conductivity of monolayer and bilayer graphene at finite densities based on resonant scatterers Phys. Rev. B 83, 165402 (2011)

19. Triozon, F., Roche, S.: Efficient linear scaling method for computing the Landauer-Büttiker conductance. Eur. Phys. J. B 46, 427 (2005)

20. Liu M.-H., Richter, K.: Efficient quantum transport simulation for bulk graphene heterojunctions. Phys. Rev. B 86115445 (2012)

21. Datta, S.: Electronic Transport in Mesoscopic Systems. Cambridge University Press, Cambridge (1996)

22. Ferry, D.K., Goodnick, S.M.: Transport in Nanostructures. Cambridge University Press, Cambridge (1997)

23. Landauer, R.: Spatial variation of currents and fields due to localized scatterers in metallic conduction. IBM J. Res. Develop. 32, 306 (1988)

24. Caroli, C., Combescot, R., Nozieres, P., Saint-James, D.: Direct calculation of the tunneling current. J. Phys. C 4, 916 (1971)

25. Lee, P.A., Fisher, D.S.: Anderson localization in two dimensions. Phys. Rev. Lett. 47, 882 (1981)

26. Fisher, D. S., Lee, P. A.: Relation between conductivity and transmission matrix. Phys. Rev. B 23, 6851 (1981)

27. Sajjad, R.N., Polanco, C., Ghosh, A.W.: Atomistic deconstruction of current flow in graphene based hetero-junctions. arXiv:1302.4473 (2013)

28. Meir, Y., Wingreen, N.S.: Landauer formula for the current through an interacting electron region. Phys. Rev. Lett. 68, 2512 (1992)

29. Hernández, A., Apel V.M., Pinheiro, F.A., Lewenkopf, C.H.: Quantum electronic transport: Linear and nonlinear conductance from the Keldysh approach. Physica A 385, 148 (2007)
30. Lewenkopf, C.H., Mucciolo, E.R., Castro Neto, A.H.: Numerical studies of conductivity and Fano factor in disordered graphene. Phys. Rev. B 77, 081410R (2008)

31. Haug, H., Jauho A.-P.: Quantum kinetics in transport and optics of semiconductors, 2nd Edition. Springer, Heidelberg (2008)

32. Mucciolo, E.R.: unpublished

33. Schomerus, H.: Effective contact model for transport through weakly-doped graphene. Phys. Rev. B 76, 045433 (2007)

34. Areshkin, D. A., Nikolić, B. K.: $I-V$ curve signatures of nonequilibrium-driven band gap collapse in magnetically ordered zigzag graphene nanoribbon two-terminal devices. Phys. Rev. B 79, 205430 (2009)

35. Lopez Sancho M.P., Lopez Sancho J.M., Rubio J.: Highly convergent schemes for the calculation of bulk and surface Green functions. J. Phys. F: Met. Phys. 15, 851 (1985)

36. Umerski, A.: Closed-form solutions to surface Green's functions. Phys. Rev. B 55, 5266 (1997)

37. Rocha, A.R., García-Suárez, V.M., Bailey, S., Lambert, C., Ferrer, J., Sanvito S.: Spin and molecular electronics in atomically generated orbital landscapes. Phys. Rev. B 73, 085414 (2006)

38. Wimmer, M.: Quantum transport in nanostructures: From computational concepts to spintronics in graphene and magnetic tunnel junctions. http://epub.uni-regensburg.de/12142/, Dissertation, University Regensburg (2009)

39. Tworzydło, J., Trauzettel, B., Titov, M., Rycerz, A., Beenakker, C.W.J.: Sub-poissonian shot noise in graphene. Phys. Rev. Lett. 96, 246802 (2006); supplementary material is found in condmat/0603315.

40. Rycerz, A., Tworzydło, J., Beenakker, C.W.J.: Anomalously large conductance fluctuations in weakly disordered graphene. Europhys. Lett. 79, 57003 (2007)

41. Metalidis, G., Bruno, P.: Green's function technique for studying electron flow in two-dimensional mesoscopic samples. Phys. Rev. B 72, 235304 (2005)

42. Todorov, T. N.: Tight-binding simulation of current-carrying nanostructures. J. Phys.: Condens. Matter 14, 3049 (2002).

43. Cresti, A., Farchioni, R., Grosso, G., Parravicini, G. P.: KeldyshGreen function formalism for current profiles in mesoscopic systems. Phys. Rev. B 68, 075306 (2003)

44. Yazyev, O.: Emergence of magnetism in graphene materials and nanostructures. Rep. Prog. Phys. 73, 056501 (2010)

45. Son, Y.W., Cohen, M.L., Louie, S.G.: Energy gaps in graphene nanoribbons. Phys. Rev. Lett. 97, 216803 (2006)

46. Xue, Y., Datta, S., Ratner, M.A.: First-principles based matrix Green's function approach to molecular electronic devices: general formalism. Chem. Phys. 281, 151 (2002)

47. Areshkin, D. A., Nikolić, B. K.: Electron density and transport in top-gated graphene nanoribbon devices: First-principles Green function algorithms for systems containing a large number of atoms. Phys. Rev. B 81, 155540 (2010)

48. Bruus H., Flensberg, K.: Many-Body Quantum Theory in Condensed Matter Physics: An Introduction. Oxford University Press, New York (2004)

49. Nazarov, Y. V., Blanter, Y. M.: Quantum Transport: Introduction to Nanoscience. Cambridge University Press, New York (2009)

50. Büttiker, M.: Coherent and sequential tunneling in series barriers. IBM J. Res. Developm. 32, 63 (1988)

51. D'Amato, J.L., Pastawski, H.M.: Conductance of a disordered linear chain including inelastic scattering events. Phys. Rev. B 41, 7411 (1990)

52. Martin, J., Akerman, N., Ulbricht, G., Lohmann, T., Smet, J.H., von Klitzing, K., Yacoby, A.: Observation of electron-hole puddles in graphene using a scanning single electron transistor. Nature Phys. 4, 144 (2008)

53. Zhang, Y., Brar, V.W., Girit, C., Zettl, A., Crommie, M.F.: Origin of spatial charge inhomogeneity in graphene. Nature Phys. 5, 722 (2009) 
54. Ishigami, M., Chen, J.H., Cullen, W.G., Fuhrer, M.S., Williams, E.D.: Atomic structure of graphene on $\mathrm{SiO}_{2}$. Nano Lett. 7, 1643 (2007)

55. Meyer, J.C., Geim, A.K., Katsnelson, M.I., Novoselov, K.S., Obergfell, D., Roth, S., Girit, Ç., Zettl, A.: On the roughness of single- and bi-layer graphene membranes. Solid State Comm. 143, 101 (2007)

56. Levy, N., Burke, S.A. Meaker, K.L., Panlasigui, M., Zettl, A., Guinea, F., Castro Neto, A.H., Crommie, M.F.: Strain-induced pseudo-magnetic fields greater than 300 Tesla in graphene nanobubbles. Science 329, 544 (2010)

57. Chen, J.H., Jang, C., Adam, S., Fuhrer, M.S., Williams, E.D., Ishigami, M.: Charged-impurity scattering in graphene. Nature Phys. 4, 377 (2008)

58. Chen, J.-H., Cullen, W. G., Jang, C., Fuhrer, M. S., Williams, E. D.: Defect scattering in graphene. Phys. Rev. Lett. 102, 236805 (2009)

59. Han, M., Brant, J.C., Kim, P.: Electron transport in disordered graphene nanoribbons. Phys. Rev. Lett. 104, 056801 (2010)

60. Peres, N.M.R.: Colloquium: The transport properties of graphene: An introduction. Rev. Mod. Phys. 82, 2673 (2010)

61. Das Sarma, S., Adam, S., Hwang, E. H., Rossi, E.: Electronic transport in two-dimensional graphene. Rev. Mod. Phys. 83, 407 (2011)

62. Adam, S., Brouwer, P.W., Das Sarma, S.: Crossover from quantum to Boltzmann transport in graphene. Phys. Rev. B 79, 201404 (2009)

63. Kłos, J.W., Zozoulenko, I.V.: Effect of short- and long range scattering on the conductivity of graphene: Boltzmann approach vs tight-binding calculations. Phys. Rev. B 82, 081414(R) (2010)

64. Pereira V.M, Castro Neto, A. H., Peres, N.M.R.: Tight-binding approach to uniaxial strain in graphene. Phys. Rev. B 80, 045401 (2009)

65. Landau, L.D., Lifshitz, E.M: Theory of elasticity, 3rd edition. Pergamon, London (1986)

66. Kim, E.-A., Castro Neto A. H.: Graphene as an electronic membrane. Europhys. Lett. 84, 57007 (2008)

67. Vozmediano, M.A.H., Katsnelson, M.I., Guinea, F.: Gauge fields in graphene. Phys. Rep. 496, 109 (2010)

68. Peierls, R.E.: Zur Theorie des Diamagnetismus von Leitungselektronen. Z. Phys. 80, 763 (1933)

69. Lundeberg, M.B., Folk, J.A.: Rippled graphene in an in-plane magnetic field: effects of a random vector potential. Phys. Rev. Lett. 105, 146804 (2010)

70. Sols, F., Guinea, F., Castro Neto A.H.: Coulomb blockade in graphene nanoribbons. Phys. Rev. Lett. 99, 166803 (2007)

71. Vérges, J.A., Guinea, F., Chiappe, G., Louis, E.: Transport regimes in surface disordered graphene sheets. Phys. Rev. B 75, 085440 (2007)

72. Evaldsson, M., Zozoulenko, I.V., Xu, H., Heinzel, T.: Edge disorder induced Anderson localization and conduction gap in graphene nanoribbons. Phys. Rev. B 78, 161407(R) 2008

73. Mucciolo, E.R., Castro Neto, A.H, Lewenkopf, C.H.: Conductance quantization and transport gaps in disordered graphene nanoribbons. Phys. Rev. B 79, 075407 (2009)

74. Brey L., Fertig, H.A.: Electronic states of graphene nanoribbons studied with the Dirac equation. Phys. Rev. B 73, 235411 (2006)

75. Zârbo, L.P., Nikolić, B.K.: Spatial distribution of local currents of massless Dirac fermions in quantum transport through graphene nanoribbons. EPL 80, 47001 (2007)

76. Chang P.-H., Nikolić, B.K.: Edge currents and nanopore arrays in zigzag and chiral graphene nanoribbons as a route toward high-ZT thermoelectrics. Phys. Rev. B 86, 041406(R) (2012)

77. Tao, C., Jiao, L., Yazyev, O.V., Chen, Y.-C., Feng, J., Zhang, X., Capaz, R.B., Tour, J.M., Zettl, A., Louie, S.G., Dai, H., Crommie, M.F.: Spatially resolving edge states of chiral graphene nanoribbons. Nature Phys. 7, 616 (2011)
78. Wakabayashi, K., Takane, Y., Sigrist, M.: Perfectly conducting channel and universality crossover in disordered graphene nanoribbons. Phys. Rev. Lett. 99, 036601 (2007)

79. Wakabayashi, K., Takane, Y.,Yamamoto, M., Sigrist, M.: Electronic transport properties of graphene nanoribbons. New J. Phys. 11, 095016 (2009)

80. Lima, L.R.F., Pinheiro, F.A., Capaz, R.B., Lewenkopf, C.H., Mucciolo, E.R.: Effects of disorder range and electronic energy on the perfect transmission in graphene nanoribbons. Phys. Rev. B 86, 205111 (2012) 